\title{
On the Spectral Asymptotics of Operators on Manifolds with Ends
}

\author{
Sandro Coriasco and Lidia Maniccia \\ Dipartimento di Matematica, Università degli Studi di Torino, V. C. Alberto, n. 10, I-10123 Torino, Italy \\ Correspondence should be addressed to Sandro Coriasco; sandro.coriasco@unito.it
}

Received 28 September 2012; Accepted 16 December 2012

Academic Editor: Changxing Miao

Copyright (C) 2013 S. Coriasco and L. Maniccia. This is an open access article distributed under the Creative Commons Attribution License, which permits unrestricted use, distribution, and reproduction in any medium, provided the original work is properly cited.

We deal with the asymptotic behaviour, for $\lambda \rightarrow+\infty$, of the counting function $N_{P}(\lambda)$ of certain positive self-adjoint operators $P$ with double order $(m, \mu), m, \mu>0, m \neq \mu$, defined on a manifold with ends $M$. The structure of this class of noncompact manifolds allows to make use of calculi of pseudodifferential operators and Fourier integral operators associated with weighted symbols globally defined on $\mathbb{R}^{n}$. By means of these tools, we improve known results concerning the remainder terms of the Weyl Formulae for $N_{P}(\lambda)$ and show how their behaviour depends on the ratio $m / \mu$ and the dimension of $M$.

\section{Introduction}

The aim of this paper is to study the asymptotic behaviour, for $\lambda \rightarrow+\infty$, of the counting function

$$
N_{P}(\lambda)=\sum_{\lambda_{j} \leq \lambda} 1,
$$

where $\lambda_{1} \leq \lambda_{2} \leq \cdots$ is the sequence of the eigenvalues, repeated according to their multiplicities, of a positive order, self-adjoint, classical, elliptic SG-pseudodifferential operator $P$ on a manifold with ends. Explicitly, SG-pseudodifferential operators $P=p(x, D)=\mathrm{Op}(p)$ on $\mathbb{R}^{n}$ can be defined via the usual left-quantization

$$
\mathrm{Pu}(x)=\frac{1}{(2 \pi)^{n}} \int e^{i x \cdot \xi} p(x, \xi) \widehat{u}(\xi) d \xi, \quad u \in \mathcal{S}\left(\mathbb{R}^{n}\right)
$$

starting from symbols $p(x, \xi) \in C^{\infty}\left(\mathbb{R}^{n} \times \mathbb{R}^{n}\right)$ with the property that, for arbitrary multiindices $\alpha, \beta$, there exist constants $C_{\alpha \beta} \geq 0$ such that the estimates

$$
\left|D_{\xi}^{\alpha} D_{x}^{\beta} p(x, \xi)\right| \leq C_{\alpha \beta}\langle\xi\rangle^{m-|\alpha|}\langle x\rangle^{\mu-|\beta|}
$$

hold for fixed $m, \mu \in \mathbb{R}$ and all $(x, \xi) \in \mathbb{R}^{n} \times \mathbb{R}^{n}$, where $\langle y\rangle=\sqrt{1+|y|^{2}}, y \in \mathbb{R}^{n}$. Symbols of this type belong to the class denoted by $S^{m, \mu}\left(\mathbb{R}^{n}\right)$, and the corresponding operators constitute the class $L^{m, \mu}\left(\mathbb{R}^{n}\right)=\mathrm{Op}\left(S^{m, \mu}\left(\mathbb{R}^{n}\right)\right)$. In the sequel we will sometimes write $S^{m, \mu}$ and $L^{m, \mu}$, respectively, fixing once and for all the dimension of the (noncompact) base manifold to $n$.

These classes of operators, introduced on $\mathbb{R}^{n}$ by Cordes [1] and Parenti [2], see also Melrose [3] and Shubin [4], form a graded algebra, that is, $L^{r, \rho} \circ L^{m, \mu} \subseteq L^{r+m, \rho+\mu}$. The remainder elements are operators with symbols in $S^{-\infty,-\infty}\left(\mathbb{R}^{n}\right)=$ $\bigcap_{(m, \mu) \in \mathbb{R}^{2}} S^{m, \mu}\left(\mathbb{R}^{n}\right)=\delta\left(\mathbb{R}^{2 n}\right)$; that is, those having kernel in $\mathcal{S}\left(\mathbb{R}^{2 n}\right)$, continuously mapping $\mathcal{S}^{\prime}\left(\mathbb{R}^{n}\right)$ to $\mathcal{S}\left(\mathbb{R}^{n}\right)$. An operator $P=\mathrm{Op}(p) \in L^{m, \mu}$ and its symbol $p \in S^{m, \mu}$ are called SG-elliptic if there exists $R \geq 0$ such that $p(x, \xi)$ is invertible for $|x|+|\xi| \geq R$ and

$$
p(x, \xi)^{-1}=O\left(\langle\xi\rangle^{-m}\langle x\rangle^{-\mu}\right)
$$

In such case we will usually write $P \in E L^{m, \mu}$. Operators in $L^{m, \mu}$ act continuously from $\mathcal{S}\left(\mathbb{R}^{n}\right)$ to itself and extend as continuous operators from $\mathcal{S}^{\prime}\left(\mathbb{R}^{n}\right)$ to itself and from 
$H^{s, \sigma}\left(\mathbb{R}^{n}\right)$ to $H^{s-m, \sigma-\mu}\left(\mathbb{R}^{n}\right)$, where $H^{s, \sigma}\left(\mathbb{R}^{n}\right), s, \sigma \in \mathbb{R}$, denotes the weighted Sobolev space

$$
\begin{gathered}
H^{s, \sigma}\left(\mathbb{R}^{n}\right)=\left\{u \in \mathcal{S}^{\prime}\left(\mathbb{R}^{n}\right):\|u\|_{s, \sigma}=\left\|\mathrm{Op}\left(\pi_{s, \sigma}\right) u\right\|_{L^{2}}<\infty\right\}, \\
\pi_{s, \sigma}(x, \xi)=\langle\xi\rangle^{s}\langle x\rangle^{\sigma} .
\end{gathered}
$$

Continuous inclusions $H^{s, \sigma}\left(\mathbb{R}^{n}\right) \hookrightarrow H^{r, \rho}\left(\mathbb{R}^{n}\right)$ hold when $s \geq r$ and $\sigma \geq \rho$, compact when both inequalities are strict, and

$$
\mathcal{S}\left(\mathbb{R}^{n}\right)=\bigcap_{(s, \sigma) \in \mathbb{R}^{2}} H^{s, \sigma}\left(\mathbb{R}^{n}\right), \quad \mathcal{S}^{\prime}\left(\mathbb{R}^{n}\right)=\bigcup_{(s, \sigma) \in \mathbb{R}^{2}} H^{s, \sigma}\left(\mathbb{R}^{n}\right) .
$$

An elliptic SG-operator $P \in L^{m, \mu}$ admits a parametrix $E \epsilon$ $L^{-m,-\mu}$ such that

$$
P E=I+K_{1}, \quad E P=I+K_{2},
$$

for suitable $K_{1}, K_{2} \in L^{-\infty,-\infty}=\mathrm{Op}\left(S^{-\infty,-\infty}\right)$, and it turns out to be a Fredholm operator. In 1987, Schrohe [5] introduced a class of noncompact manifolds, the so-called SG-manifolds, on which it is possible to transfer from $\mathbb{R}^{n}$ the whole SG-calculus. In short, these are manifolds which admit a finite atlas whose changes of coordinates behave like symbols of order $(0,1)$ (see [5] for details and additional technical hypotheses). The manifolds with cylindrical ends are a special case of SG-manifolds, on which also the concept of SG-classical operator makes sense; moreover, the principal symbol of an SG-classical operator $P$ on a manifold with cylindrical ends $M$, in this case a triple $\sigma(P)=$ $\left(\sigma_{\psi}(P), \sigma_{e}(P), \sigma_{\psi e}(P)\right)=\left(p_{\psi}, p_{e}, p_{\psi e}\right)$, has an invariant meaning on $M$, see Egorov and Schulze [6], Maniccia and Panarese [7], Melrose [3], and Section 2. We indicate the subspaces of classical symbols and operators adding the subscript ${ }_{\mathrm{cl}}$ to the notation introduced above.

The literature concerning the study of the eigenvalue asymptotics of elliptic operators is vast and covers a number of different situations and operator classes, see, for example, the monograph by Ivrii [8]. Then, we only mention a few of the many existing papers and books on this deeply investigated subject, which are related to the case we consider here, either by the type of symbols and underlying spaces, or by the techniques which are used. We refer the reader to the corresponding reference lists for more complete informations. On compact manifolds, well-known results were proved by Hörmander [9] and Guillemin [10], see also the book by Kumano-go [11]. On the other hand, for operators globally defined on $\mathbb{R}^{n}$, see Boggiatto et al. [12], Helffer [13], Hörmander [14], Mohammed [15], Nicola [16], and Shubin [4]. Many other situations have been considered, see the cited book by Ivrii. On manifolds with ends, Christiansen and Zworski [17] studied the Laplace-Beltrami operator associated with a scattering metric, while Maniccia and Panarese [7] applied the heat kernel method to study operators similar to those considered here.

Here we deal with the case of manifolds with ends for $P \in E L_{\mathrm{cl}}^{m, \mu}(M)$, positive and self-adjoint, such that $m, \mu>0, m \neq \mu$, focusing on the (invariant) meaning of the constants appearing in the corresponding Weyl formulae and on achieving a better estimate of the remainder term. Note that the situation we consider here is different from that of the Laplace-Beltrami operator investigated in [17], where continuous spectrum is present as well. In fact, in view of Theorem 14, $\operatorname{spec}(P)$ consists only of a sequence of real isolated eigenvalues $\left\{\lambda_{j}\right\}$ with finite multiplicity.

As recalled above, a first result concerning the asymptotic behaviour of $N_{P}(\lambda)$ for operators including those considered in this paper was proved by Maniccia and Panarese in [7], giving, for $\lambda \rightarrow+\infty$,

$$
N_{P}(\lambda)= \begin{cases}C_{1} \lambda^{n / m}+o\left(\lambda^{n / m}\right) & \text { for } m<\mu, \\ C_{0}^{1} \lambda^{n / m} \log \lambda+o\left(\lambda^{n / m} \log \lambda\right) & \text { for } m=\mu, \\ C_{2} \lambda^{n / \mu}+o\left(\lambda^{n / \mu}\right) & \text { for } m>\mu\end{cases}
$$

Note that the constants $C_{1}, C_{2}, C_{0}^{1}$ above depend only on the principal symbol of $P$, which implies that they have an invariant meaning on the manifold $M$, see Sections 2 and 3. On the other hand, in view of the technique used there, the remainder terms appeared in the form $o\left(\lambda^{n / \min \{m, \mu\}}\right)$ and $o\left(\lambda^{n / m} \log \lambda\right)$ for $m \neq \mu$ and $m=\mu$, respectively. An improvement in this direction for operators on $\mathbb{R}^{n}$ had been achieved by Nicola [16], who, in the case $m=\mu$, proved that

$$
N_{P}(\lambda)=C_{0}^{1} \lambda^{n / m} \log \lambda+O\left(\lambda^{n / m}\right), \quad \lambda \longrightarrow+\infty,
$$

while, for $m \neq \mu$, showed that the remainder term has the form $O\left(\lambda^{(n / \min \{m, \mu\})-\varepsilon}\right)$ for a suitable $\varepsilon>0$. A further improvement of these results in the case $m=\mu$ has recently appeared in Battisti and Coriasco [18], where it has been shown that, for a suitable $\varepsilon>0$,

$$
\begin{array}{r}
N_{P}(\lambda)=C_{0}^{1} \lambda^{n / m} \log \lambda+C_{0}^{2} \lambda^{n / m}+O\left(\lambda^{(n / m)-\varepsilon}\right), \\
\lambda \longrightarrow+\infty .
\end{array}
$$

Even the constant $C_{0}^{2}$ has an invariant meaning on $M$, and both $C_{0}^{1}$ and $C_{0}^{2}$ are explicitly computed in terms of trace operators defined on $L_{\mathrm{cl}}^{m, m}(M)$.

In this paper the remainder estimates in the case $m \neq \mu$ are further improved. More precisely, we first consider the power $Q=P^{1 / \max \{m, \mu\}}$ of $P$ (see Maniccia et al. [19] for the properties of powers of SG-classical operators). Then, by studying the asymptotic behaviour in $\lambda$ of the trace of the operator $\widehat{\psi}_{\lambda}(-Q)$, $\psi_{\lambda}(t)=\psi(t) e^{-i t \lambda}, \psi \in C_{0}^{\infty}(\mathbb{R})$, defined via a Spectral Theorem and approximated in terms of Fourier Integral Operators, we prove the following.

Theorem 1. Let $M$ be a manifold with ends of dimension $n$ and let $P \in E L_{c l}^{m, \mu}(M)$ be a positive self-adjoint operator such that 
$m, \mu>0, m \neq \mu$, with domain $H^{m, \mu}(M) \hookrightarrow L^{2}(M)$. Then, the following Weyl formulae hold for $\lambda \rightarrow+\infty$ :

$$
N_{P}(\lambda)=\left\{\begin{array}{c}
C_{1} \lambda^{n / m}+O\left(\lambda^{n / \mu}\right)+O\left(\lambda^{(n / m)-(1 / \mu)}\right) \\
=C_{1} \lambda^{n / m}+O\left(\lambda^{(n / m)-\varepsilon_{1}}\right) \quad \text { for } m<\mu, \\
C_{2} \lambda^{n / \mu}+O\left(\lambda^{n / m}\right)+O\left(\lambda^{(n / \mu)-(1 / m)}\right) \\
=C_{2} \lambda^{n / \mu}+O\left(\lambda^{(n / \mu)-\varepsilon_{2}}\right) \quad \text { for } m>\mu,
\end{array}\right.
$$

where $\varepsilon_{1}=\min \{1 / \mu, n((1 / m)-(1 / \mu))\}$ and $\varepsilon_{2}=\min \{1 /$ $m, n((1 / \mu)-(1 / m))\}$.

The order of the remainder is then determined by the ratio of $m$ and $\mu$ and the dimension of $M$, since

$$
\begin{aligned}
& \frac{n}{m}-\frac{1}{\mu} \leq \frac{n}{\mu}, \quad \text { for } m<\mu \Longleftrightarrow 1<\frac{\mu}{m} \leq 1+\frac{1}{n}, \\
& \frac{n}{\mu}-\frac{1}{m} \leq \frac{n}{m}, \quad \text { for } m>\mu \Longleftrightarrow 1<\frac{m}{\mu} \leq 1+\frac{1}{n} .
\end{aligned}
$$

In particular, when $\max \{m, \mu\} / \min \{m, \mu\} \geq 2$, the remainder is always $O\left(\lambda^{n / \max \{m, \mu\}}\right)$.

Examples include operators of Schrödinger type on $M$, that is, $P=-\Delta_{g}+V, \Delta_{g}$ the Laplace-Beltrami operator in $M$ associated with a suitable metric $g, V$ a smooth potential that, in the local coordinates $x \in U_{N} \subseteq \mathbb{R}^{n}$ on the cylindrical end growths as $\langle x\rangle^{\mu}$, with an appropriate $\mu>0$ related to $g$. Such examples will be discussed in detail, together with the sharpness of the results in Theorem 1, in the forthcoming paper [20], see also [21].

The key point in the proof of Theorem 1 is the study of the asymptotic behaviour for $\lambda \rightarrow+\infty$ of integrals of the form

$$
I(\lambda)=\int e^{i(-t \lambda+\varphi(t ; x, \xi)-x \xi)} \psi(t) a(t ; x, \xi) d t d \xi d x,
$$

where $a$ and $\varphi$ satisfy certain growth conditions in $x$ and $\xi$ (see Section 3 for more details). The integrals $I(\lambda)$ represent in fact the local expressions of the trace of $\widehat{\psi}_{\lambda}(-Q)$, obtained through the so-called "geometric optic method," specialised to the SG situation, see, for example, Coriasco [22, 23], Coriasco and Rodino [24]. To treat the integrals $I(\lambda)$ we proceed similarly to Grigis and Sjöstrand [25], Helffer and Robert [26], see also Tamura [27].

The paper is organised as follows. Section 2 is devoted to recall the definition of SG-classical operators on a manifold with ends $M$. In Section 3 we show that the asymptotic behaviour of $N_{P}(\lambda), \lambda \rightarrow+\infty$, for a positive self-adjoint operator $P \in L_{\mathrm{cl}}^{m, \mu}(M), m, \mu>0$, is related to the asymptotic behaviour of oscillatory integrals of the form $I(\lambda)$. In Section 4 we conclude the proof of Theorem 1, investigating the behaviour of $I(\lambda)$ for $\lambda \rightarrow+\infty$. Finally, some technical details are collected in the Appendix.

\section{SG-Classical Operators on Manifolds with Ends}

From now on, we will be concerned with the subclass of SGoperators given by those elements $P \in L^{m, \mu}\left(\mathbb{R}^{n}\right),(m, \mu) \in$ $\mathbb{R}^{2}$, which are SG-classical, that is, $P=\mathrm{Op}(p)$ with $p \in$ $S_{\mathrm{cl}}^{m, \mu}\left(\mathbb{R}^{n}\right) \subset S^{m, \mu}\left(\mathbb{R}^{n}\right)$. We begin recalling the basic definitions and results (see, e.g., $[6,19]$ for additional details and proofs).

Definition 2. (i) A symbol $p(x, \xi)$ belongs to the class $S_{\mathrm{cl}(\xi)}^{m, \mu}\left(\mathbb{R}^{n}\right)$ if there exist $p_{m-i, \cdot}(x, \xi) \in \widetilde{\mathscr{H}}_{\xi}^{m-i}\left(\mathbb{R}^{n}\right), i=0,1, \ldots$, positively homogeneous functions of order $m-i$ with respect to the variable $\xi$, smooth with respect to the variable $x$, such that, for a 0 -excision function $\omega$,

$$
\begin{array}{r}
p(x, \xi)-\sum_{i=0}^{N-1} \omega(\xi) p_{m-i, .}(x, \xi) \in S^{m-N, \mu}\left(\mathbb{R}^{n}\right), \\
N=1,2, \ldots .
\end{array}
$$

(ii) A symbol $p(x, \xi)$ belongs to the class $S_{\mathrm{cl}(x)}^{m, \mu}\left(\mathbb{R}^{n}\right)$ if there exist $p_{\cdot, \mu-k}(x, \xi) \in \widetilde{\mathscr{H}}_{x}^{\mu-k}\left(\mathbb{R}^{n}\right), k=0, \ldots$, positively homogeneous functions of order $\mu-k$ with respect to the variable $x$, smooth with respect to the variable $\xi$, such that, for a 0 -excision function $\omega$,

$$
\begin{array}{r}
p(x, \xi)-\sum_{k=0}^{N-1} \omega(x) p_{\cdot, \mu-k}(x, \xi) \in S^{m, \mu-N}\left(\mathbb{R}^{n}\right), \\
N=1,2, \ldots .
\end{array}
$$

Definition 3. A symbol $p(x, \xi)$ is SG-classical, and we write $p \in S_{\mathrm{cl}(x, \xi)}^{m, \mu}\left(\mathbb{R}^{n}\right)=S_{\mathrm{cl}}^{m, \mu}\left(\mathbb{R}^{n}\right)=S_{\mathrm{cl}}^{m, \mu}$, if

(i) there exist $p_{m-j,}(x, \xi) \in \widetilde{\mathscr{H}}_{\xi}^{m-j}\left(\mathbb{R}^{n}\right)$ such that for a 0 excision function $\omega, \omega(\xi) p_{m-j,}(x, \xi) \in S_{\mathrm{cl}(x)}^{m-j, \mu}\left(\mathbb{R}^{n}\right)$ and

$$
\begin{array}{r}
p(x, \xi)-\sum_{j=0}^{N-1} \omega(\xi) p_{m-j,}(x, \xi) \in S^{m-N, \mu}\left(\mathbb{R}^{n}\right), \\
N=1,2, \ldots ;
\end{array}
$$

(ii) there exist $p_{\cdot, \mu-k}(x, \xi) \in \widetilde{\mathscr{H}}_{x}^{\mu-k}\left(\mathbb{R}^{n}\right)$ such that for a 0 excision function $\omega, \omega(x) p_{\cdot, \mu-k}(x, \xi) \in S_{\mathrm{cl}(\xi)}^{m, \mu-k}\left(\mathbb{R}^{n}\right)$ and

$$
p(x, \xi)-\sum_{k=0}^{N-1} \omega(x) p_{\cdot, \mu-k} \in S^{m, \mu-N}\left(\mathbb{R}^{n}\right), \quad N=1,2, \ldots
$$

We set $L_{\mathrm{cl}(x, \xi)}^{m, \mu}\left(\mathbb{R}^{n}\right)=L_{\mathrm{cl}}^{m, \mu}=\mathrm{Op}\left(S_{\mathrm{cl}}^{m, \mu}\right)$.

Remark 4. The definition could be extended in a natural way from operators acting between scalars to operators acting between (distributional sections of) vector bundles. One should then use matrix-valued symbols whose entries satisfy the estimates (3).

Note that the definition of SG-classical symbol implies a condition of compatibility for the terms of the expansions 
with respect to $x$ and $\xi$. In fact, defining $\sigma_{\psi}^{m-j}$ and $\sigma_{e}^{\mu-i}$ on $S_{\mathrm{cl}(\xi)}^{m, \mu}$ and $S_{\mathrm{cl}(x)}^{m, \mu}$, respectively, as

$$
\begin{gathered}
\sigma_{\psi}^{m-j}(p)(x, \xi)=p_{m-j, \cdot}(x, \xi), \quad j=0,1, \ldots \\
\sigma_{e}^{\mu-i}(p)(x, \xi)=p_{\cdot, \mu-i}(x, \xi), \quad i=0,1, \ldots
\end{gathered}
$$

It is possibile to prove that

$$
\begin{aligned}
p_{m-j, \mu-i} & =\sigma_{\psi e}^{m-j, \mu-i}(p)=\sigma_{\psi}^{m-j}\left(\sigma_{e}^{\mu-i}(p)\right) \\
& =\sigma_{e}^{\mu-i}\left(\sigma_{\psi}^{m-j}(p)\right), \quad j=0,1, \ldots, i=0,1, \ldots
\end{aligned}
$$

Moreover, the composition of two SG-classical operators is still classical. For $P=\mathrm{Op}(p) \in L_{\mathrm{cl}}^{m, \mu}$ the triple $\sigma(P)=$ $\left(\sigma_{\psi}(P), \sigma_{e}(P), \sigma_{\psi e}(P)\right)=\left(p_{m,}, p_{\cdot, \mu}, p_{m, \mu}\right)=\left(p_{\psi}, p_{e}, p_{\psi e}\right)$ is called the principal symbol of $P$. The three components are also called the $\psi$-, $e$ - and $\psi e$-principal symbol, respectively. This definition keeps the usual multiplicative behaviour, that is, for any $R \in L_{\mathrm{cl}}^{r, \rho}, S \in L_{\mathrm{cl}}^{s, \sigma},(r, \rho),(s, \sigma) \in \mathbb{R}^{2}, \sigma(R S)=$ $\sigma(S) \sigma(T)$, with component-wise product in the right-hand side. We also set

$$
\begin{aligned}
\operatorname{Sym}_{p}(P)(x, \xi)= & \operatorname{Sym}_{p}(p)(x, \xi) \\
= & p_{\mathbf{m}}(x, \xi) \\
= & \omega(\xi) p_{\psi}(x, \xi) \\
& +\omega(x)\left(p_{e}(x, \xi)-\omega(\xi) p_{\psi e}(x, \xi)\right),
\end{aligned}
$$

for a fixed 0 -excision function $\omega$. Theorem 5 allows to express the ellipticity of SG-classical operators in terms of their principal symbol.

Theorem 5. An operator $P \in L_{c l}^{m, \mu}$ is elliptic if and only if each element of the triple $\sigma(P)$ is nonvanishing on its domain of definition.

As a consequence, denoting by $\left\{\lambda_{j}\right\}$ the sequence of eigenvalues of $P$, ordered such that $j \leq k \Rightarrow \lambda_{j} \leq \lambda_{k}$, with each eigenvalue repeated accordingly to its multiplicity, the counting function $N_{P}(\lambda)=\sum_{\lambda_{j} \leq \lambda} 1$ is well defined for a SG-classical elliptic self-adjoint operator $P$ see, for example, $[16,18,20,21]$. We now introduce the class of noncompact manifolds with which we will deal.

Definition 6. A manifold with a cylindrical end is a triple $(M, X,[f])$, where $M=\mathscr{M} \coprod_{C} \mathscr{C}$ is a $n$-dimensional smooth manifold and

(i) $\mathscr{M}$ is a smooth manifold, given by $\mathscr{M}=\left(M_{0} \backslash D\right) \cup$ $C$ with a $n$-dimensional smooth compact manifold without boundary $M_{0}, D$ a closed disc of $M_{0}$, and $C \subset D$ a collar neighbourhood of $\partial D$ in $M_{0}$;

(ii) $\mathscr{C}$ is a smooth manifold with boundary $\partial \mathscr{C}=X$, with $X$ diffeomorphic to $\partial D$;

(iii) $f:\left[\delta_{f}, \infty\right) \times \mathbb{S}^{n-1} \rightarrow \mathscr{C}, \delta_{f}>0$, is a diffeomorphism, $f\left(\left\{\delta_{f}\right\} \times \mathbb{S}^{n-1}\right)=X$ and $f\left(\left\{\left[\delta_{f}, \delta_{f}+\varepsilon_{f}\right)\right\} \times \mathbb{S}^{n-1}\right)$, $\varepsilon_{f}>0$, is diffeomorphic to $C$; (iv) the symbol $\bigsqcup_{C}$ means that we are gluing $\mathscr{M}$ and $\mathscr{C}$, through the identification of $C$ and $f\left(\left\{\left[\delta_{f}, \delta_{f}+\varepsilon_{f}\right)\right\} \times\right.$ $\left.\mathbb{S}^{n-1}\right)$;

(v) the symbol $[f]$ represents an equivalence class in the set of functions

$\left\{g:\left[\delta_{g}, \infty\right) \times \mathbb{S}^{n-1} \longrightarrow \mathscr{C}: g\right.$ is a diffeomorphism,

$g\left(\left\{\delta_{g}\right\} \times \mathbb{S}^{n-1}\right)=X$ and $g\left(\left[\delta_{g}, \delta_{g}+\varepsilon_{g}\right) \times \mathbb{S}^{n-1}\right)$,

$\varepsilon_{g}>0$, is diffeomorphic to $\left.C\right\}$,

where $f \sim g$ if and only if there exists a diffeomorphism $\Theta \in$ $\operatorname{Diff}\left(\mathbb{S}^{n-1}\right)$ such that

$$
\left(g^{-1} \circ f\right)(\rho, \gamma)=(\rho, \Theta(\gamma)),
$$

for all $\rho \geq \max \left\{\delta_{f}, \delta_{g}\right\}$ and $\gamma \in \mathbb{S}^{n-1}$.

We use the following notation:

(i) $U_{\delta_{f}}=\left\{x \in \mathbb{R}^{n}:|x|>\delta_{f}\right\}$;

(ii) $\mathscr{C}_{\tau}=f\left([\tau, \infty) \times \mathbb{S}^{n-1}\right)$, where $\tau \geq \delta_{f}$. The equivalence condition (22) implies that $\mathscr{C}_{\tau}$ is well defined;

(iii) $\pi: \mathbb{R}^{n} \backslash\{0\} \rightarrow(0, \infty) \times \mathbb{S}^{n-1}: x \mapsto \pi(x)=$ $(|x|, x /|x|)$;

(iv) $f_{\pi}=f \circ \pi: \overline{U_{\delta_{f}}} \rightarrow \mathscr{C}$ is a parametrisation of the end. Let us notice that, setting $F=g_{\pi}^{-1} \circ f_{\pi}$, the equivalence condition (22) implies

$$
F(x)=|x| \Theta\left(\frac{x}{|x|}\right) .
$$

We also denote the restriction of $f_{\pi}$ mapping $U_{\delta_{f}}$ onto $\dot{\mathscr{C}}=\mathscr{C} \backslash X$ by $\dot{f}_{\pi}$.

The couple $\left(\dot{\mathscr{C}}, \dot{f}_{\pi}^{-1}\right)$ is called the exit chart. If $\mathscr{A}=$ $\left\{\left(\Omega_{i}, \psi_{i}\right)\right\}_{i=1}^{N}$ is such that the subset $\left\{\left(\Omega_{i}, \psi_{i}\right)\right\}_{i=1}^{N-1}$ is a finite atlas for $\mathscr{M}$ and $\left(\Omega_{N}, \psi_{N}\right)=\left(\dot{\mathscr{C}}, \dot{f}_{\pi}^{-1}\right)$, then $M$, with the atlas $\mathscr{A}$, is a SG-manifold (see [4]). An atlas $\mathscr{A}$ of such kind is called admissible. From now on, we restrict the choice of atlases on $M$ to the class of admissible ones. We introduce the following spaces, endowed with their natural topologies,

$$
\begin{gathered}
\mathcal{S}\left(U_{\delta}\right)=\left\{u \in C^{\infty}\left(U_{\delta}\right): \forall \alpha, \beta \in \mathbb{N}^{n}\right. \\
\left.\forall \delta^{\prime}>\delta \sup _{x \in U_{\delta^{\prime}}}\left|x^{\alpha} \partial^{\beta} u(x)\right|<\infty\right\}, \\
\mathcal{S}_{0}\left(U_{\delta}\right)=\bigcap_{\delta^{\prime} \searrow \delta}\left\{u \in \mathcal{S}\left(\mathbb{R}^{n}\right): \operatorname{supp} u \subseteq \overline{U_{\delta^{\prime}}}\right\}, \\
\mathcal{S}(M)=\left\{u \in C^{\infty}(M): u \circ \dot{f}_{\pi} \in \mathcal{S}\left(U_{\delta_{f}}\right)\right. \\
\text { for any exit map } \left.f_{\pi}\right\}, \\
\mathcal{S}^{\prime}(M) \text { denotes the dual space of } \mathcal{S}(M) .
\end{gathered}
$$


Definition 7. The set $S^{m, \mu}\left(U_{\delta_{f}}\right)$ consists of all the symbols $a \in C^{\infty}\left(U_{\delta_{f}}\right)$ which fulfill (3) for $(x, \xi) \in U_{\delta_{f}} \times \mathbb{R}^{n}$ only. Moreover, the symbol $a$ belongs to the subset $\mathrm{SG}_{\mathrm{cl}}^{m, \mu}\left(U_{\delta_{f}}\right)$ if it admits expansions in asymptotic sums of homogeneous symbols with respect to $x$ and $\xi$ as in Definitions 2 and 3, where the remainders are now given by SG-symbols of the required order on $U_{\delta_{f}}$.

Note that, since $U_{\delta_{f}}$ is conical, the definition of homogeneous and classical symbol on $U_{\delta_{f}}$ makes sense. Moreover, the elements of the asymptotic expansions of the classical symbols can be extended by homogeneity to smooth functions on $\mathbb{R}^{n} \backslash\{0\}$, which will be denoted by the same symbols. It is a fact that, given an admissible atlas $\left\{\left(\Omega_{i}, \psi_{i}\right)\right\}_{i=1}^{N}$ on $M$, there exists a partition of unity $\left\{\theta_{i}\right\}$ and a set of smooth functions $\left\{\chi_{i}\right\}$ which are compatible with the SG-structure of $M$, that is,

(i) $\operatorname{supp} \theta_{i} \subset \Omega_{i}$, supp $\chi_{i} \subset \Omega_{i}, \chi_{i} \theta_{i}=\theta_{i}, i=1, \ldots, N$;

(ii) $\left|\partial^{\alpha}\left(\theta_{N} \circ \dot{f}_{\pi}\right)(x)\right| \leq C_{\alpha}\langle x\rangle^{-|\alpha|}$ and $\left|\partial^{\alpha}\left(\chi_{N} \circ \dot{f}_{\pi}\right)(x)\right| \leq$ $C_{\alpha}\langle x\rangle^{-|\alpha|}$ for all $x \in U_{\delta_{f}}$.

Moreover, $\theta_{N}$ and $\chi_{N}$ can be chosen so that $\theta_{N} \circ \dot{f}_{\pi}$ and $\chi_{N} \circ \dot{f}_{\pi}$ are homogeneous of degree 0 on $U_{\delta}$. We denote by $u^{*}$ the composition of $u: \psi_{i}\left(\Omega_{i}\right) \subset \mathbb{R}^{n} \rightarrow \mathbb{C}$ with the coordinate patches $\psi_{i}$, and by $v_{*}$ the composition of $v: \Omega_{i} \subset M \rightarrow \mathbb{C}$ with $\psi_{i}^{-1}, i=1, \ldots, N$. It is now possible to give the definition of SG-pseudodifferential operator on $M$.

Definition 8. Let $M$ be a manifold with a cylindrical end. A linear operator $P: \mathcal{S}(M) \rightarrow \mathcal{S}^{\prime}(M)$ is an SGpseudodifferential operator of order $(m, \mu)$ on $M$, and we write $P \in L^{m, \mu}(M)$, if, for any admissible atlas $\left\{\left(\Omega_{i}, \psi_{i}\right)\right\}_{i=1}^{N}$ on $M$ with exit chart $\left(\Omega_{N}, \psi_{N}\right)$ :

(1) for all $i=1, \ldots, N-1$ and any $\theta_{i}, \chi_{i} \in C_{c}^{\infty}\left(\Omega_{i}\right)$, there exist symbols $p^{i}(x, \xi) \in S^{m}\left(\psi_{i}\left(\Omega_{i}\right)\right)$ such that

$$
\begin{array}{r}
\left(\chi_{i} P \theta_{i} u^{*}\right)_{*}(x)=\iint e^{i(x-y) \cdot \xi} p^{i}(x, \xi) u(y) d y d x, \\
u \in C^{\infty}\left(\psi_{i}\left(\Omega_{i}\right)\right) ;
\end{array}
$$

(2) for any $\theta_{N}, \chi_{N}$ of the type described above, there exists a symbol $p^{N}(x, \xi) \in \mathrm{SG}^{m, \mu}\left(U_{\delta_{f}}\right)$ such that

$$
\begin{array}{r}
\left(\chi_{N} P \theta_{N} u^{*}\right)_{*}(x)=\iint e^{i(x-y) \cdot \xi} p^{N}(x, \xi) u(y) d y d x \\
u \in \mathcal{S}_{0}\left(U_{\delta_{f}}\right)
\end{array}
$$

(3) $K_{P}$, the Schwartz kernel of $P$, is such that

$$
K_{P} \in C^{\infty}((M \times M) \backslash \Delta) \bigcap \mathcal{S}((\dot{\mathscr{C}} \times \dot{\mathscr{C}}) \backslash W),
$$

where $\Delta$ is the diagonal of $M \times M$ and $W=\left(\dot{f}_{\pi} \times\right.$ $\left.\dot{f}_{\pi}\right)(V)$ with any conical neighbourhood $V$ of the diagonal of $U_{\delta_{f}} \times U_{\delta_{f}}$.
The most important local symbol of $P$ is $p^{N}$. Our definition of SG-classical operator on $M$ differs slightly from the one in [7].

Definition 9. Let $P \in L^{m, \mu}(M) . P$ is an SG-classical operator on $M$, and we write $P \in L_{\mathrm{cl}}^{m, \mu}(M)$, if $p^{N}(x, \xi) \in S_{\mathrm{cl}}^{m, \mu}\left(U_{\delta_{f}}\right)$ and the operator $P$, restricted to the manifold $\mathscr{M}$, is classical in the usual sense.

The usual homogeneous principal symbol $p_{\psi}$ of an SGclassical operator $P \in L_{\mathrm{cl}}^{m, \mu}(M)$ is of course well defined as a smooth function on $T^{*} M$. In order to give an invariant definition of the principal symbols homogeneous in $x$ of an operator $P \in L_{\mathrm{cl}}^{m, \mu}(M)$, the subbundle $T_{X}^{*} M=\{(x, \xi) \in$ $\left.T^{*} M: x \in X, \xi \in T_{x}^{*} M\right\}$ was introduced. The notions of ellipticity can be extended to operators on $M$ as well.

Definition 10. Let $P \in L_{\mathrm{cl}}^{m, \mu}(M)$ and let us fix an exit map $f_{\pi}$. We can define local objects $p_{m-j, \mu-i}, p_{\cdot, \mu-i}$ as

$$
\begin{aligned}
p_{m-j, \mu-i}(\theta, \xi) & =p_{m-j, \mu-i}^{N}(\theta, \xi), \quad \theta \in \mathbb{S}^{n-1}, \xi \in \mathbb{R}^{n} \backslash\{0\} \\
p_{\cdot, \mu-i}(\theta, \xi) & =p_{\cdot, \mu-i}^{N}(\theta, \xi), \quad \theta \in \mathbb{S}^{n-1}, \xi \in \mathbb{R}^{n}
\end{aligned}
$$

Definition 11. An operator $P \in L_{\mathrm{cl}}^{m, \mu}(M)$ is elliptic, and we write $P \in E L_{\mathrm{cl}}^{m, \mu}(M)$, if the principal part of $p^{N} \in S^{m, \mu}\left(U_{\delta_{f}}\right)$ satisfies the SG-ellipticity conditions on $U_{\delta_{f}} \times \mathbb{R}^{n}$ and the operator $P$, restricted to the manifold $\mathscr{M}$, is elliptic in the usual sense.

Proposition 12. The properties $P \in L^{m, \mu}(M)$ and $P \in$ $L_{c l}^{m, \mu}(M)$, as well as the notion of SG-ellipticity, do not depend on the (admissible) atlas on M. Moreover, the local functions $p_{e}$ and $p_{\psi e}$ give rise to invariantly defined elements of $C^{\infty}\left(T_{X}^{*} M\right)$ and $C^{\infty}\left(T_{X}^{*} M \backslash 0\right)$, respectively.

Then, with any $P \in L_{\mathrm{cl}}^{m, \mu}(M)$, it is associated an invariantly defined principal symbol in three components $\sigma(P)=\left(p_{\psi}, p_{e}, p_{\psi e}\right)$. Finally, through local symbols given by $\pi_{s, \sigma}^{j}(x, \xi)=\langle\xi\rangle^{s}, j=1, \ldots, N-1$, and $\pi_{s, \sigma}^{N}(x, \xi)=\langle\xi\rangle^{s}\langle x\rangle^{\sigma}$, $s, \sigma \in \mathbb{R}$, we get a SG-elliptic operator $\prod_{s, \sigma} \in L_{\text {cl }}^{s, \sigma}(M)$ and introduce the (invariantly defined) weighted Sobolev spaces $H^{s, \sigma}(M)$ as

$$
H^{s, \sigma}(M)=\left\{u \in \mathcal{S}^{\prime}(M): \prod_{s, \sigma} u \in L^{2}(M)\right\}
$$

The properties of the spaces $H^{s, \sigma}\left(\mathbb{R}^{n}\right)$ extend to $H^{s, \sigma}(M)$ without any change, as well as the continuity of the linear mappings $P: H^{s, \sigma}(M) \rightarrow H^{s-m, \sigma-\mu}(M)$ induced by $P \in$ $L^{m, \mu}(M)$, mentioned in Section 1 . 


\section{Spectral Asymptotics for SG-Classical Elliptic Self-Adjoint Operators on Manifolds with Ends}

In this section we illustrate the procedure to prove Theorem 1 , similar to $[13,25,27]$. The result will follow from the Trace formula (39), (41), the asymptotic behaviour (42), and the Tauberian Theorem 19. The remaining technical points, in particular the proof of the asymptotic behaviour of the integrals appearing in (41), are described in Section 4 and in the Appendix.

Let the operator $P \in E L_{\mathrm{cl}}^{m, \mu}(M)$ be considered as an unbounded operator $P: \mathcal{S}(M) \subset H^{0,0}(M)=L^{2}(M) \rightarrow$ $L^{2}(M)$. The following proposition can be proved by reducing to the local situation and using continuity and ellipticity of $P$, its parametrix, and the density of $\delta(M)$ in the $H^{s, \sigma}(M)$ spaces.

Proposition 13. Every $P \in E L_{c l}^{m, \mu}(M)$, considered as an unbounded operator $P: \mathcal{S}(M) \subset L^{2}(M) \rightarrow L^{2}(M)$, admits a unique closed extension, still denoted by $P$, whose domain is $\mathscr{D}(P)=H^{m, \mu}(M)$.

From now on, when we write $P \in E L_{\mathrm{cl}}^{m, \mu}(M)$ we always mean its unique closed extension, defined in Proposition 13. As standard, we denote by $\varrho(P)$ the resolvent set of $P$, that is, the set of all $\lambda \in \mathbb{C}$ such that $\lambda I-P$ maps $H^{m, \mu}(M)$ bijectively onto $L^{2}(M)$. The spectrum of $P$ is then $\operatorname{spec}(P)=\mathbb{C} \backslash \varrho(P)$. The next theorem was proved in [7].

Theorem 14 (Spectral theorem). Let $P \in E L_{c l}^{m, \mu}(M)$ be regarded as a closed unbounded operator on $L^{2}(M)$ with dense domain $H^{m, \mu}(M)$. Assume also that $m, \mu>0$ and $P^{*}=P$. Then

(i) $(\lambda I-P)^{-1}$ is a compact operator on $L^{2}(M)$ for every $\lambda \in \varrho(P)$. More precisely, $(\lambda I-P)^{-1}$ is an extension by continuity from $\mathcal{S}(M)$ or a restriction from $\mathcal{S}^{\prime}(M)$ of an operator in $E L_{c l}^{-m,-\mu}(M)$.

(ii) spec $(P)$ consists of a sequence of real isolated eigenvalues $\left\{\lambda_{j}\right\}$ with finite multiplicity, clustering at infinity; the orthonormal system of eigenfunctions $\left\{e_{j}\right\}_{j \geq 1}$ is complete in $L^{2}(M)=H^{0,0}(M)$. Moreover, $e_{j} \in \mathcal{S}(M)$ for all $j$.

Given a positive self-adjoint operator $P \in E L_{\mathrm{cl}}^{m, \mu}(M)$, $m, \mu>0, \mu \neq m$, we can assume, without loss of generality (considering, if necessary, $P+c$ in place of $P$, with $c \in \mathbb{R}$ a suitably large constant), $1 \leq \lambda_{1} \leq \lambda_{2}$... Define the counting function $N_{P}(\lambda), \lambda \in \mathbb{R}$, as

$$
N_{P}(\lambda)=\sum_{\lambda_{j} \leq \lambda} 1=\#(\operatorname{spec}(P) \cap(-\infty, \lambda]) .
$$

Clearly, $N_{P}$ is nondecreasing, continuous from the right and supported in $[0,+\infty)$. If we set $Q=P^{1 / l}, l=\max \{m, \mu\}$ (see [19] for the definition of the powers of $P$ ), $Q$ turns out to be a SG-classical elliptic self-adjoint operator with
$\sigma(Q)=\left(p_{\psi}^{1 / l}, p_{e}^{1 / l}, p_{\psi e}^{1 / l}\right)$. We denote by $\left\{\eta_{j}\right\}$ the sequence of eigenvalues of $Q$, which satisfy $\eta_{j}=\lambda_{j}^{1 / l}$. We can then, as above, consider $N_{Q}(\eta)$. It is a fact that $N_{Q}(\eta)=O\left(\eta^{n / l}\right)$, see [7].

From now on we focus on the case $\mu>m>0$. The case $m>\mu>0$ can be treated in a completely similar way, exchanging the role of $x$ and $\xi$. So we can start from a closed positive self-adjoint operator $Q \in E L_{\mathrm{cl}}^{m, 1}(M)$ with domain $\mathscr{D}(Q)=H^{m, 1}(M), m \in(0,1)$. For $u \in H^{m, 1}(M), t \in \mathbb{R}$, we set

$$
U(t) u=\sum_{j=1}^{\infty} e^{i t \eta_{j}}\left(u, e_{j}\right)_{L^{2}(M)} e_{j}
$$

and the series converges in the $L^{2}(M)$ norm (cf., e.g., [25]). Clearly, for all $t \in \mathbb{R}, U(t)$ is a unitary operator such that

$$
U(0)=I, \quad U(t+s)=U(t) U(s), \quad t, s \in \mathbb{R} .
$$

Moreover, if $u \in H^{k m, k}(M)$ for some $k \in \mathbb{N}, U(t) u \in$ $C^{k}\left(\mathbb{R}, H^{0,0}(M)\right) \cap \cdots \cap C^{0}\left(\mathbb{R}, H^{k m, k}(M)\right)$ and, for $u \in$ $H^{m, 1}(M)$, we have $D_{t} U(t) u-Q U(t) u=0, U(0) u=u$, which implies that $v(t, x)=U(t) u(x)$ is a solution of the Cauchy problem

$$
\left(D_{t}-Q\right) v=0,\left.\quad v\right|_{t=0}=u \text {. }
$$

Let us fix $\psi \in \mathcal{S}(\mathbb{R})$. We can then define the operator $\widehat{\psi}(-Q)$ either by using the formula

$$
\widehat{\psi}(-Q) u=\sum_{j=1}^{\infty} \widehat{\psi}\left(-\eta_{j}\right)\left(u, e_{j}\right)_{L^{2}(M)} e_{j},
$$

or by means of the vector-valued integral $\left(\int \psi(t) U(t) d t\right) u=$ $\int \psi(t) U(t) u d t, u \in H^{0,0}(M)$. Indeed, there exists $N_{0} \in \mathbb{N}$ such that $\sum_{j=1}^{\infty} \eta_{j}^{-N_{0}}<\infty$, so the definition makes sense and gives an operator in $\mathscr{L}\left(L^{2}(M)\right)$ with norm bounded by $\|\psi\|_{L^{1}(\mathbb{R})}$. The following lemma, whose proof can be found in the Appendix, is an analog on $M$ of Proposition 1.10.11 in [13].

Lemma 15. $\widehat{\psi}(-Q)$ is an operator with kernel $K_{\psi}(x, y)=$ $\sum_{j} \widehat{\psi}\left(-\eta_{j}\right) e_{j}(x) \overline{e_{j}(y)} \in \mathcal{S}(M \times M)$.

Clearly, we then have

$$
\int_{M} K_{\psi}(x, x) d x=\sum_{j} \widehat{\psi}\left(-\eta_{j}\right) .
$$

By the analysis in [22-24, 28] (see also [29]), the above Cauchy problem (33) can solve modulo $\delta(M)$ by means of a smooth family of operators $V(t)$, defined for $t \in(-T, T)$, $T>0$ suitably small, in the sense that $\left(D_{t}-Q\right) \circ V$ is a family of smoothing operators and $V(0)$ is the identity on $\mathcal{S}^{\prime}(M)$. More explicitly, the following theorem holds (see the Appendix for some details concerning the extension to the manifold $M$ of the results on $\mathbb{R}^{n}$ proved in $\left.[22-24,28]\right)$. 
Theorem 16. Define $V(t) u=\sum_{k=1}^{N} \chi_{k} A_{k}(t)\left(\theta_{k} u\right)$, where $\theta_{k}$ and $\chi_{k}$ are as in Definition 8, with $\chi_{k} \theta_{k}=\theta_{k}, k=1, \ldots, N$, while the $A_{k}(t)$ are SG FIOs which, in the local coordinate open set $U_{k}=\psi_{k}\left(\Omega_{k}\right)$ and with $v \in \mathcal{S}\left(\mathbb{R}^{n}\right)$, are given by

$$
\left(A_{k}(t) v\right)(x)=\int e^{i \varphi_{k}(t ; x, \xi)} a_{k}(t ; x, \xi) \widehat{v}(\xi) d \xi
$$

Each $A_{k}(t)$ solves a local Cauchy problem $\left(D_{t}-Q_{k}\right) \circ A_{k} \epsilon$ $C^{\infty}\left((-T, T), L^{-\infty,-\infty}\left(\mathbb{R}^{n}\right)\right), A_{k}(0)=I$, with $Q_{k}=O p\left(q_{k}\right)$ and $\left\{q_{k}\right\} \subset S G_{c l}^{m, 1}\left(\mathbb{R}^{n}\right)$ local (complete) symbol of $Q$ associated with $\left\{\theta_{k}\right\},\left\{\chi_{k}\right\}$, with phase and amplitude functions such that

$$
\begin{gathered}
\partial_{t} \varphi_{k}(t ; x, \xi)-q_{k}\left(x, d_{x} \varphi_{k}(t ; x, \xi)\right)=0, \quad \varphi_{k}(0 ; x, \xi)=x \xi, \\
a_{k} \in C^{\infty}\left((-T, T), S G_{c l}^{0,0}\left(\mathbb{R}^{n}\right)\right), \quad a_{k}(0 ; x, \xi)=1 .
\end{gathered}
$$

Then, $V(t)$ satisfies

$$
\left(D_{t}-Q\right) \circ V \in C^{\infty}\left((-T, T), L^{-\infty,-\infty}(M)\right), \quad V(0)=I
$$

and $U-V \in C^{\infty}\left((-T, T), L^{-\infty,-\infty}(M)\right)$.

Remark 17. Trivially, for $k=1, \ldots, N-1, q_{k}$ and $a_{k}$ can be considered SG-classical, since, in those cases, they actually have order $-\infty$ with respect to $x$, by the fact that $q_{k}(x, \xi)$ vanishes for $x$ outside a compact set.

Remark 18. Notation like $b \in C^{\infty}\left((-T, T), S^{r, \rho}\left(\mathbb{R}^{n}\right)\right), B \in$ $C^{\infty}\left((-T, T), L^{r, \rho}(M)\right)$, and similar, in Theorem 16 and in the sequel, also mean that the seminorms of the involved elements in the corresponding spaces (induced, in the mentioned cases, by (3)), are uniformly bounded with respect to $t \in(-T, T)$.

If we write $\psi_{\lambda}(t)=\psi(t) e^{-i t \lambda}$ in place of $\psi(t)$, for a chosen $\psi \in C_{0}^{\infty}((-T, T))$, the trace formula (35) becomes

$$
\int_{M} K_{\psi_{\lambda}}(x, x) d x=\sum \widehat{\psi}\left(\lambda-\eta_{j}\right)
$$

Let us denote the kernel of $U-V$ by $r(t ; x, y) \quad \epsilon$ $C^{\infty}((-T, T), \delta(M \times M))$. Then, the distribution kernel of $\int e^{-i t \lambda} \psi(t) U(t) d t=\widehat{\psi}_{\lambda}(-Q)$ is

$$
\begin{aligned}
K_{\psi_{\lambda}}(x, y)= & \sum_{k=1}^{N} \chi_{k}(x) \iint \psi(t) e^{i\left(-t \lambda+\varphi_{k}(t ; x, \xi)-y \xi\right)} \\
& \times a_{k}(t ; x, \xi) d t d \xi \theta_{k}(y) \\
& +\int e^{-i t \lambda} \psi(t) r(t ; x, y) d t
\end{aligned}
$$

where the local coordinates in the right-hand side depend on $k$ and, to simplify the notation, we have omitted the corresponding coordinate maps. By the choices of $\psi, \theta_{k}$ and $\chi_{k}$ we obtain

$$
\begin{aligned}
\sum_{j} \widehat{\psi}\left(\lambda-\eta_{j}\right)= & \sum_{k=1}^{N} \iiint \psi(t) e^{i\left(-t \lambda+\varphi_{k}(t ; x, \xi)-x \xi\right)} \\
& \times a_{k}(t ; x, \xi) \theta_{k}(x) d t d \xi d x \\
& +\iint e^{-i t \lambda} \psi(t) r(t ; x, x) d t d x \\
= & \sum_{k=1}^{N} \iiint \psi(t) e^{i\left(-t \lambda+\varphi_{k}(t ; x, \xi)-x \xi\right)} \\
& \times a_{k}(t ; x, \xi) \theta_{k}(x) d t d \xi d x \\
& +O\left(|\lambda|^{-\infty}\right) .
\end{aligned}
$$

Let $\psi \in C_{0}^{\infty}((-T, T)), T>0$, be such that $\psi(0)=1$ and $\widehat{\psi} \geq 0, \widehat{\psi}(0)>0$ (e.g., set $\psi=\chi * \overline{\bar{\chi}}$ with a suitable $\chi \epsilon$ $\left.C_{0}^{\infty}((-T, T))\right)$. By the analysis of the asymptotic behaviour of the integrals appearing in (41), described in Section 4, we finally obtain

$$
\sum_{j} \widehat{\psi}\left(\lambda-\eta_{j}\right)= \begin{cases}\frac{n}{m} d_{0} \lambda^{(n / m)-1}+O\left(\lambda^{n^{*}-1}\right) \\ \text { for } \lambda \longrightarrow+\infty \\ O\left(|\lambda|^{-\infty}\right) & \text { for } \lambda \longrightarrow-\infty\end{cases}
$$

with $n^{*}=\min \{n,(n / m)-1\}$. The following Tauberian theorem is a slight modification of Theorem 4.2 .5 of [13] (see the Appendix).

Theorem 19. Assume that

(i) $\psi \in C_{0}^{\infty}(\mathbb{R})$ is an even function satisfying $\psi(0)=1$, $\widehat{\psi} \geq 0, \widehat{\psi}(0)>0$;

(ii) $N_{Q}(\lambda)$ is a nondecreasing function, supported in $[0,+\infty)$, continuous from the right, with polynomial growth at infinity and isolated discontinuity points of first kind $\left\{\eta_{j}\right\}, j \in \mathbb{N}$, such that $\eta_{j} \rightarrow+\infty$;

(iii) there exists $d_{0} \geq 0$ such that

$$
\begin{aligned}
\sum_{j} \widehat{\psi}\left(\lambda-\eta_{j}\right) & =\int \widehat{\psi}(\lambda-\eta) d N_{Q}(\eta) \\
& = \begin{cases}\frac{n}{m} d_{0} \lambda^{(n / m)-1}+O\left(\lambda^{n^{*}-1}\right) \\
O\left(|\lambda|^{-\infty}\right) & \text { for } \lambda \longrightarrow+\infty,\end{cases}
\end{aligned}
$$

with $m \in(0,1), n^{*}=\min \{n,(n / m)-1\}$.

Then

$$
N_{\mathrm{Q}}(\lambda)=\frac{d_{0}}{2 \pi} \lambda^{n / m}+O\left(\lambda^{n^{*}}\right), \quad \text { for } \lambda \longrightarrow+\infty .
$$


Remark 20. The previous statement can be modified as follows: with $\psi, N_{Q}$, and $m$ as in Theorem 19 , when

$$
\begin{aligned}
& \int \widehat{\psi}(\lambda-\eta) d N_{Q}(\eta) \\
& \quad= \begin{cases}\frac{n}{m} d_{0} \lambda^{(n / m)-1}+O\left(\lambda^{(n / m)-2}\right)+O\left(\lambda^{n-1}\right) \\
O\left(|\lambda|^{-\infty}\right) & \text { for } \lambda \longrightarrow+\infty,\end{cases} \\
&
\end{aligned}
$$

with $m \in(0,1)$, then $N_{Q}(\lambda)=\left(d_{0} / 2 \pi\right) \lambda^{n / m}+O\left(\lambda^{(n / m)-1}\right)+$ $O\left(\lambda^{n}\right)$, for $\lambda \rightarrow+\infty$.

\section{Proof of Theorem 1}

In view of Theorem 19 and Remark 20, to complete the proof of Theorem 1 we need to show that (42) holds. To this aim, as explained previously, this section will be devoted to studying the asymptotic behaviour for $|\lambda| \rightarrow+\infty$ of

$$
I(\lambda)=\int e^{i \Phi(t ; x, \xi ; \lambda)} \psi(t) a(t ; x, \xi) d t d \xi d x,
$$

where $\psi \in C_{0}^{\infty}((-T, T)), \psi(0)=1, a \in C^{\infty}((-T, T)$, $\left.S^{0,0}\left(\mathbb{R}^{n}\right)\right), a(0 ; x, \xi)=1$, and

$$
\begin{array}{r}
\Phi(t ; x, \xi ; \lambda)=\varphi(t ; x, \xi)-x \xi-t \lambda, \\
\varphi \in C^{\infty}\left((-T, T), S_{\mathrm{cl}}^{1,1}\left(\mathbb{R}^{n}\right)\right),
\end{array}
$$

such that

(i) $\partial_{t} \varphi(t ; x, \xi)=q\left(x, d_{x} \varphi(t ; x, \xi)\right), \varphi(0 ; x, \xi)=x \xi$;

(ii) $C^{-1}\langle\xi\rangle \leq\left\langle d_{x} \varphi(t ; x, \xi)\right\rangle \leq C\langle\xi\rangle$, for a suitable constant $C>1$;

(iii) $q \in S_{\mathrm{cl}}^{m, 1}\left(\mathbb{R}^{n}\right), 0<m<1$, SG-elliptic.

Since $q^{-1}(x, \xi) \in O\left(\langle x\rangle^{-1}\langle\xi\rangle^{-m}\right)$ for $|x|+|\xi| \geq R>0$, it is not restrictive to assume that this estimate holds on the whole phase space, so that, for a certain constant $A>1$,

$$
A^{-1}\langle x\rangle\langle\xi\rangle^{m} \leq q(x, \xi) \leq A\langle x\rangle\langle\xi\rangle^{m} .
$$

Remark 21. The assumption on $q^{-1}$ above amounts, at most, to modifying $q$ by adding and subtracting a compactly supported symbol, that is, an element of $S^{-\infty,-\infty}\left(\mathbb{R}^{n}\right)$. The corresponding solutions $\varphi$ and $a$ of the eikonal and transport equations, respectively, would then change, at most, by an element of $C^{\infty}\left((-T, T), S^{-\infty,-\infty}\left(\mathbb{R}^{n}\right)\right)$, see $[23,24,28]$. It is immediate, by integration by parts with respect to $t$, that an integral as (46) is $O\left(|\lambda|^{-\infty}\right)$ for $a \in C^{\infty}\left((-T, T), S^{-\infty,-\infty}\left(\mathbb{R}^{n}\right)\right)$. Then, the modified $q$ obviously keeps the same sign everywhere.

For two functions $f, g$, defined on a common subset $X$ of $\mathbb{R}^{d_{1}}$ and depending on parameters $y \in Y \subseteq \mathbb{R}^{d_{2}}$, we will write $f \prec g$ or $f(x, y) \prec g(x, y)$ to mean that there exists a suitable constant $c>0$ such that $|f(x, y)| \leq c|g(x, y)|$ for all $(x, y) \in X \times Y$. The notation $f \sim g$ or $f(x, y) \sim g(x, y)$ means that both $f \prec g$ and $g \prec f$ hold.
Remark 22. The ellipticity of $q$ yields, for $\lambda<0$,

$$
\left.\partial_{t} \Phi(t ; x, \xi ; \lambda)=q\left(x, d_{x} \varphi(t ; x, \xi)\right)-\lambda\right\rangle\langle x\rangle\langle\xi\rangle^{m}+|\lambda|
$$

which, by integration by parts, implies $I(\lambda)=O\left(|\lambda|^{-\infty}\right)$ when $\lambda \rightarrow-\infty$.

From now on any asymptotic estimate is to be meant for $\lambda \rightarrow+\infty$.

We will make use of a partition of unity on the phase space. The supports of its elements will depend on suitably large positive constants $k_{1}, k_{2}>1$. We also assume, as it is possible, $\lambda \geq \lambda_{0}$, again with an appropriate $\lambda_{0} \gg 1$. As we will see below, the values of $k_{1}, k_{2}$, and $\lambda_{0}$ depend only on $q$ and its associated seminorms.

Proposition 23. Let $H_{1}$ be any function in $C_{0}^{\infty}(\mathbb{R})$ such that $\operatorname{supp} H_{1} \subseteq\left[\left(2 k_{1}\right)^{-1}, 2 k_{1}\right], 0 \leq H_{1} \leq 1$ and $H_{1} \equiv 1$ on $\left[k_{1}^{-1}, k_{1}\right]$, where $k_{1}>1$ is a suitably chosen, large positive constant. Then

$$
\begin{gathered}
I(\lambda)=O\left(\lambda^{-\infty}\right)+\int e^{i \Phi(t ; x, \xi ; \lambda)} \psi(t) H_{1}\left(\frac{\langle x\rangle\langle\xi\rangle^{m}}{\lambda}\right) \\
\times a(t ; x, \xi) d t d \xi d x .
\end{gathered}
$$

Proof. Write

$$
\begin{gathered}
I(\lambda)=\int e^{i \Phi(t ; x, \xi ; \lambda)} \psi(t)\left[1-H_{1}\left(\frac{\langle x\rangle\langle\xi\rangle^{m}}{\lambda}\right)\right] \\
\quad \times a(t ; x, \xi) d t d \xi d x \\
+\int e^{i \Phi(t ; x, \xi ; \lambda)} \psi(t) H_{1}\left(\frac{\langle x\rangle\langle\xi\rangle^{m}}{\lambda}\right) \\
\times a(t ; x, \xi) d t d \xi d x,
\end{gathered}
$$

and observe that, by $A^{-1}\langle x\rangle\langle\xi\rangle^{m} \leq q(x, \xi) \leq A\langle x\rangle\langle\xi\rangle^{m}$, $x, \xi \in \mathbb{R}^{n}$, we find

$$
\begin{gathered}
\left|\partial_{t} \Phi(t ; x, \xi ; \lambda)\right| \geq \frac{\lambda}{2}+\left(\frac{k_{1}}{2}-A C\right)\langle x\rangle\langle\xi\rangle^{m} \\
\text { when } \frac{\langle x\rangle\langle\xi\rangle^{m}}{\lambda} \leq k_{1}^{-1}, \\
\left|\partial_{t} \Phi(t ; x, \xi ; \lambda)\right| \geq \frac{(A C)^{-1}}{2}\langle x\rangle\langle\xi\rangle^{m}+\left[\frac{(A C)^{-1}}{2} k_{1}-1\right] \lambda \\
\text { when } \frac{\langle x\rangle\langle\xi\rangle^{m}}{\lambda} \geq k_{1} .
\end{gathered}
$$

Thus, if $k_{1}>2 A C$ we have $\left|\partial_{t} \Phi(t ; x, \xi ; \lambda)\right| \sim \lambda+\langle x\rangle\langle\xi\rangle^{m}$ on the support of $1-H_{1}\left(\langle x\rangle\langle\xi\rangle^{m} / \lambda\right)$, and the assertion follows integrating by parts with respect to $t$ in the first integral of (51).

Remark 24. We actually choose $k_{1}>4 A C>2 A C$, since this will be needed in the proof of Proposition 28; see also Section $\mathrm{C}$ in the Appendix. 
Let us now pick $H_{2} \in C_{0}^{\infty}(\mathbb{R})$ such that $0 \leq H_{2}(v) \leq 1$, $H_{2}(v)=1$ for $|v| \leq k_{2}$ and $H_{2}(v)=0$ for $|v| \geq 2 k_{2}$, where $k_{2}>1$ is a constant which we will choose big enough (see below). We can then write

$$
\begin{aligned}
(\lambda)= & O\left(\lambda^{-\infty}\right)+\int e^{i \Phi(t ; x, \xi ; \lambda)} \psi(t) H_{1}\left(\frac{\langle x\rangle\langle\xi\rangle^{m}}{\lambda}\right) \\
& \times H_{2}(|\xi|) a(t ; x, \xi) d t d \xi d x \\
+ & \int e^{i \Phi(t ; x, \xi ; \lambda)} \psi(t) H_{1}\left(\frac{\langle x\rangle\langle\xi\rangle^{m}}{\lambda}\right) \\
\times\left[1-H_{2}(|\xi|)\right] a(t ; x, \xi) d t d \xi d x & \\
= & O\left(\lambda^{-\infty}\right)+I_{1}(\lambda)+I_{2}(\lambda) .
\end{aligned}
$$

In what follows, we will systematically use the notation $S^{r, \rho}=S^{r, \rho}(y, \eta), y \in \mathbb{R}^{k}, \eta \in \mathbb{R}^{l}$, to generally denote functions depending smoothly on $y$ and $\eta$ and satisfying SG-type estimates of order $r, \rho$ in $y, \eta$. In a similar fashion, $S_{T}^{r, \rho}=C^{\infty}\left((-T, T), S^{r, \rho}(y, \eta)\right)$ will stand for some function of the same kind which, additionally, depends smoothly on $t \in(-T, T)$, and, for all $s \in \mathbb{Z}_{+}, D_{t}^{s} C^{\infty}\left((-T, T), S^{r, \rho}(y, \eta)\right)$ satisfies SG-type estimates of order $r, \rho$ in $y, \eta$, uniformly with respect to $t \in(-T, T)$.

To estimate $I_{1}(\lambda)$, we will apply the stationary phase theorem. We begin by rewriting the integral $I_{1}(\lambda)$, using the fact that $\varphi$ is solution of the eikonal equation associated with $q$ and that $q$ is a classical SG-symbol. Note that then $\partial_{t}^{2} \varphi \in$ $C^{\infty}\left((-T, T), S_{\mathrm{cl}}^{2 m-1,1}\left(\mathbb{R}^{n}\right)\right) \subseteq C^{\infty}\left((-T, T), S_{\mathrm{cl}}^{m, 1}\left(\mathbb{R}^{n}\right)\right)$, since

$$
\begin{aligned}
\partial_{t}^{2} \varphi(t ; x, \xi)=\sum_{i=1}^{n} & \left(\partial_{\xi_{i}} q\right)\left(x, d_{x} \varphi(t ; x, \xi)\right) \\
& \times \partial_{x_{i}}\left(q\left(x, d_{x} \varphi(t ; x, \xi)\right)\right) .
\end{aligned}
$$

In view of the Taylor expansion of $\varphi$ at $t=0$, recalling the property $q(x, \xi)=\omega(x) q_{e}(x, \xi)+S^{m, 0}(x, \xi), \omega$ a fixed 0 excision function, we have, for some $0<\delta_{1}<1$,

$$
\begin{aligned}
\Phi(t ; x, \xi ; \lambda)= & -\lambda t-x \xi+\varphi(0 ; x, \xi)+t \partial_{t} \varphi(0 ; x, \xi) \\
& +\frac{t^{2}}{2} \partial_{t}^{2} \varphi\left(t \delta_{1} ; x, \xi\right) \\
= & -\lambda t+t q(x, \xi)+t^{2} S_{T}^{2 m-1,1}(x, \xi) \\
= & -\lambda t+t \omega(x) q_{e}(x, \xi)+t S^{m, 0}(x, \xi) \\
& +t^{2} S_{T}^{2 m-1,1}(x, \xi) \\
= & -\lambda t+t \omega(x) q_{e}(x, \xi)+t S^{m, 0}(x, \xi) \\
& +t^{2} \omega(x) S_{T, e}^{2 m-1,1}(x, \xi)+t^{2} S_{T}^{2 m-1,0}(x, \xi),
\end{aligned}
$$

where the subscript $e$ denotes the $x$-homogeneous (exit) principal parts of the involved symbols, which are all SGclassical and real-valued, see [28].
Observe that $|x| \sim \lambda$ on the support of the integrand in $I_{1}(\lambda)$, so that we can, in fact, assume $\omega(x) \equiv 1$ there. Indeed, recalling that, by definition, $\omega \in C^{\infty}\left(\mathbb{R}^{n}\right), \omega(v) \equiv 0$ for $|v| \leq$ $B, \omega(v) \equiv 1$ for $|v| \geq 2 B$, with a fixed constant $B>0$, it is enough to observe that

$$
|\xi|<1, \quad\langle x\rangle\langle\xi\rangle^{m} \sim \lambda \Longrightarrow\langle x\rangle \sim \lambda,
$$

which of course implies $\langle x\rangle \sim|x|$, provided that $\lambda_{0} \leq \lambda$ is large enough. Moreover, by the ellipticity of $q$, writing $x=$ $|x| \varsigma, \varsigma \in \mathbb{S}^{n-1}$, with the constant $A>1$ of (48),

$$
\begin{aligned}
& A^{-1}\langle x\rangle\langle\xi\rangle^{m} \leq q(x, \xi)=\omega(x) q_{e}(x, \xi)+S^{m, 0}(x, \xi) \\
& \leq A\langle x\rangle\langle\xi\rangle^{m} \\
& \Longrightarrow A^{-1} \frac{\langle x\rangle}{|x|}\langle\xi\rangle^{m} \leq \omega(x) q_{e}(\varsigma, \xi) \\
&+\frac{S^{m, 0}(x, \xi)}{|x|} \leq A \frac{\langle x\rangle}{|x|}\langle\xi\rangle^{m} \\
& \Longrightarrow A^{-1}\langle\xi\rangle^{m} \leq q_{e}(\varsigma, \xi) \leq A\langle\xi\rangle^{m}, \\
& \varsigma \in \mathbb{S}^{n-1}, \xi \in \mathbb{R}^{n}
\end{aligned}
$$

taking the limit for $|x| \rightarrow+\infty$. Then, setting $x=\lambda \zeta \varsigma, \zeta \in$ $[0,+\infty), \varsigma \in \mathbb{S}^{n-1}, \lambda \geq \lambda_{0} \gg 1$, in $I_{1}(\lambda)$, by homogeneity and the previous remarks, we can write

$$
\begin{aligned}
\Phi(t ; \lambda \zeta \varsigma, \xi ; \lambda)= & -\lambda t+t \omega(\lambda \zeta \varsigma) q_{e}(\lambda \zeta \varsigma, \xi)+t S^{m, 0}(\lambda \zeta \varsigma, \xi) \\
& +t^{2} \omega(\lambda \zeta \varsigma) S_{T, e}^{2 m-1,1}(\lambda \zeta \varsigma, \xi) \\
& +t^{2} S_{T}^{2 m-1,0}(\lambda \zeta \varsigma, \xi) \\
= & -\lambda t+\lambda \zeta t q_{e}(\varsigma, \xi)+\lambda \zeta t^{2} S_{T, e}^{2 m-1,1}(\varsigma, \xi) \\
& +t S^{m, 0}(\lambda \zeta \varsigma, \xi)+t^{2} S_{T}^{2 m-1,0}(\lambda \zeta \varsigma, \xi) \\
= & \lambda\left[-t+\zeta t q_{e}(\varsigma, \xi)+\zeta t^{2} S_{T, e}^{2 m-1,1}(\varsigma, \xi)\right] \\
& +G_{1}(\lambda ; t, \zeta ; \varsigma, \xi) \\
= & \lambda F_{1}(t, \zeta ; \varsigma, \xi)+G_{1}(\lambda ; t, \zeta ; \varsigma, \xi),
\end{aligned}
$$

and find, in view of the compactness of the support of the integrand (see the proof of Proposition 25 below) and the hypotheses

$$
\begin{aligned}
I_{1}(\lambda)=\lambda^{n} \int e^{i \lambda F_{1}(t, \zeta \zeta ; \zeta \xi \xi} e^{i G_{1}(\lambda ; t, \zeta ; \zeta, \xi \xi} \psi(t) \\
\quad \times a(t ; \lambda \zeta \zeta, \xi) H_{1}\left(\frac{\langle\lambda \zeta\rangle\langle\xi\rangle^{m}}{\lambda}\right) \\
\quad \times H_{2}(|\xi|) \zeta^{n-1} d t d \zeta d \xi d \varsigma \\
=\frac{\lambda^{n}}{(2 \pi)^{n}} \int e^{i \lambda F_{1}(X, Y)} U_{1}(X, Y ; \lambda) d X d Y,
\end{aligned}
$$

with $X=(t, \zeta), Y=(\varsigma, \xi)$. We can now prove the following. 
Proposition 25. Choosing the constants $k_{1}, \lambda_{0}>1$ large enough and $T>0$ suitably small, one has, for any $k_{2}>1$ and for a certain sequence $c_{j}, j=0,1, \ldots$,

$$
I_{1}(\lambda) \sim \sum_{j=0}^{+\infty} c_{j} \lambda^{n-1-j}
$$

that is, $I_{1}(\lambda)=c_{0} \lambda^{n-1}+O\left(\lambda^{n-2}\right)$, with

$$
c_{0}=\frac{1}{(2 \pi)^{n-1}} \int_{\mathbb{R}^{n}} \int_{\mathbb{S}^{n-1}} \frac{H_{2}(|\xi|)}{q_{e}(\varsigma, \xi)^{n}} d \varsigma d \xi .
$$

Proof. It is easy to see that, on the support of $U_{1}$, the phase function $F_{1}(X, Y)$ admits a unique, nondegenerate, stationary point $X_{0}=X_{0}(Y)=\left(0, q_{e}(\varsigma, \xi)^{-1}\right)$, that is, $F_{1, X}^{\prime}\left(X_{0}(Y), Y\right)=$ 0 for all $Y$ such that $(X, Y) \in \operatorname{supp} U_{1}$, provided that $T>$ 0 is chosen suitably small (see, e.g., [25, page 136]), and the Hessian $\operatorname{det}\left(F_{1, X}^{\prime \prime}\left(X_{0}(Y), Y\right)\right)$ equals $-q_{e}(\varsigma, \xi)^{2}<0$. Moreover, the amplitude function

$$
\begin{aligned}
U_{1}(X, Y ; \lambda)= & \psi(t) H_{1}\left(\frac{\langle\lambda \zeta\rangle\langle\xi\rangle^{m}}{\lambda}\right) H_{2}(|\xi|) \\
& \times a(t ; \lambda \zeta \varsigma, \xi) \zeta^{n-1} e^{i G(\lambda ; t, \zeta ; \zeta, \xi)}
\end{aligned}
$$

is compactly supported with respect to the variables $X$ and $Y$ and satisfies, for all $\gamma \in \mathbb{Z}_{+}^{2}$,

$$
D_{X}^{\gamma} U_{1}(X, Y ; \lambda) \prec 1
$$

for all $X, Y, \lambda \geq \lambda_{0}$. In fact,

(1) $\psi \in C_{0}^{\infty}((-T, T)), \varsigma \in \mathbb{S}^{n-1}, \operatorname{supp}\left[H_{2}(|\xi|)\right] \subseteq\{\xi:$ $\left.|\xi| \leq 2 k_{2}\right\}$, and

$$
\begin{aligned}
\left(2 k_{1}\right)^{-1} & \leq\langle\xi\rangle^{m} \sqrt{\frac{1}{\lambda^{2}}+\zeta^{2}} \leq 2 k_{1} \\
& \Longrightarrow 0<\sqrt{\frac{1}{4 k_{1}^{2}\left\langle 2 k_{2}\right\rangle^{2 m}}-\frac{1}{\lambda_{0}^{2}}} \leq \zeta \leq 2 k_{1},
\end{aligned}
$$

where $\lambda_{0}>2 k_{1}\left\langle 2 k_{2}\right\rangle^{m}$;

(2) all the factors appearing in the expression of $U_{1}$ are uniformly bounded, together with all their $X$ derivatives, for $X \in S_{X}=\operatorname{supp} \psi \times\left[\zeta_{0}, \zeta_{1}\right], Y \in S_{Y}=$ $\mathbb{S}^{n-1} \times\left\{\xi:|\xi| \leq 2 k_{2}\right\}$, and $\lambda \geq \lambda_{0}$.

Of course, (2) trivially holds for the cutoff functions $\psi(t)$ and $H_{2}(|\xi|)$, and for the factor $\zeta^{n-1}$. Since $a(t ; x, \xi) \in$ $S_{T}^{0,0}(x, \xi)$, on $S_{X} \times S_{Y}$ we have, for all $\gamma \in \mathbb{Z}_{+}^{2}$ and $\lambda \geq \lambda_{0}>1$,

$$
\begin{aligned}
D_{X}^{\gamma} a(t ; \lambda \zeta \varsigma, \xi) \prec\langle\lambda \zeta\rangle^{-\gamma_{2}} \lambda^{\gamma_{2}}\langle\xi\rangle^{m} & \prec \frac{1}{\left(\left(1 / \lambda^{2}\right)+\zeta^{2}\right)^{\gamma_{2} / 2}} \\
& <\frac{1}{\zeta \gamma_{2}} \prec 1 .
\end{aligned}
$$

Moreover, since $G_{1} \in S_{T}^{m, 0}(x, \xi)$ is actually in $S_{T}^{-\infty, 0}(x, \xi) \subset$ $S_{T}^{0,0}(x, \xi)$ on $S_{X} \times S_{Y}$, the same holds for $\exp \left(i G_{1}\right)$, by an application of the Faà di Bruno formula for the derivatives of compositions of functions, so also this factor fulfills the desired estimates. Finally, another straightforward computation shows that, for all $\gamma_{2} \in \mathbb{Z}_{+}$and $\lambda \geq \lambda_{0}>1$,

$$
D_{\zeta}^{\gamma_{2}} H_{1}\left(\frac{\langle\lambda \zeta\rangle\langle\xi\rangle^{m}}{\lambda}\right) \prec 1
$$

on $S_{X} \times S_{Y}$. The proposition is then a consequence of the stationary phase theorem (see [30, Proposition 1.2.4], [31, Theorem 7.7.6]), applied to the integral with respect to $X=(t, \zeta)$. In particular, the leading term is given by $\lambda^{n} /(2 \pi)^{n-1}$ times the integral with respect to $Y$ of $\lambda^{-1} \mid$ det $\left.\left(F_{1, X}^{\prime \prime}\left(X_{0}(Y), Y\right)\right)\right|^{-1 / 2} U_{1}\left(X_{0}(Y), Y ; \lambda\right)$, that is

$$
\begin{aligned}
I_{1}(\lambda)=\frac{\lambda^{n-1}}{(2 \pi)^{n-1}} \int_{\mathbb{R}^{n}} \int_{\mathbb{S}^{n-1}} \frac{1}{q_{e}(\varsigma, \xi)} \psi(0) \\
\times H_{1}\left(\frac{\left\langle\lambda / q_{e}(\varsigma, \xi)\right\rangle\langle\xi\rangle^{m}}{\lambda}\right) \\
\times \frac{H_{2}(|\xi|)}{q_{e}(\varsigma, \xi)^{n-1}} a\left(0 ; \frac{\lambda \varsigma}{q_{e}(\varsigma, \xi)}, \xi\right) d \varsigma d \xi \\
+O\left(\lambda^{n-2}\right) \quad \frac{\lambda^{n-1}}{(2 \pi)^{n-1}} \int_{\mathbb{R}^{n}} \int_{\mathbb{S}^{n-1}} \quad H_{1}\left(\frac{\left\langle\lambda / q_{e}(\varsigma, \xi)\right\rangle\langle\xi\rangle^{m}}{\lambda}\right) \\
=\frac{\lambda^{n-1}}{(2 \pi)^{n-1}} \int_{\mathbb{R}^{n}} \int_{\mathbb{S}^{n-1}} \frac{H_{2}(|\xi|)}{q_{e}(\varsigma, \xi)^{n}} d \varsigma d \xi+O\left(\lambda^{n-2}\right) \\
q_{e}(\varsigma, \xi)^{n} d \varsigma d \xi+O\left(\lambda^{n-2}\right),
\end{aligned}
$$

recalling that $\psi(0)=1, a(0 ; x, \xi)=1$ for all $x, \xi \in \mathbb{R}^{n}$.

Indeed, having chosen $k_{1}>2 A, \lambda_{0}>2 k_{1}\left\langle 2 k_{2}\right\rangle^{m}$, (58) implies

$$
\begin{aligned}
k_{1}^{-1}<A^{-1} & <\frac{\left\langle\lambda / q_{e}(\varsigma, \xi)\right\rangle\langle\xi\rangle^{m}}{\lambda} \\
& =\sqrt{\left(\frac{\langle\xi\rangle^{m}}{\lambda}\right)^{2}+\left(\frac{\langle\xi\rangle^{m}}{q_{e}(\varsigma, \xi)}\right)^{2}} \\
& <\sqrt{\frac{1}{4 k_{1}^{2}}+A^{2}}<k_{1},
\end{aligned}
$$

uniformly for $\varsigma \in \mathbb{S}^{n-1}, \xi \in \operatorname{supp}\left[H_{2}(|\xi|)\right], \lambda \geq \lambda_{0}$. This concludes the proof.

Let us now consider $I_{2}(\lambda)$. We follow a procedure close to that used in the proof of Theorem 7.7.6 of [31]. However, since here we lack the compactness of the support of the amplitude with respect to $x$, we need explicit estimates to show that all the involved integrals are convergent, so we give the argument in full detail in what follows. 
We initially proceed as in the analysis of $I_{1}(\lambda)$ mentioned previously. In view of the presence of the factor $1-H_{2}(|\xi|)$ in the integrand, we can now assume $|\xi| \geq k_{2}>\max \{B, 1\}$, $B>0$ the radius of the smallest ball in $\mathbb{R}^{n}$ including $\operatorname{supp}(1-$ $\omega)$, so that $q(x, \xi)=\omega(\xi) q_{\psi}(x, \xi)+S^{m-1,1}(x, \xi)=q_{\psi}(x, \xi)+$ $S^{m-1,1}(x, \xi)$. Then, with some $0<\delta_{2}<1$,

$$
\begin{aligned}
\Phi(t ; x, \xi ; \lambda)= & -\lambda t-x \xi+\varphi(0 ; x, \xi)+t \partial_{t} \varphi(0 ; x, \xi) \\
& +\frac{t^{2}}{2} \partial_{t}^{2} \varphi\left(t \delta_{2} ; x, \xi\right) \\
= & -\lambda t+t q(x, \xi)+t^{2} S_{T}^{2 m-1,1}(x, \xi) \\
= & -\lambda t+t q_{\psi}(x, \xi)+t S^{m-1,1}(x, \xi) \\
& +t^{2} S_{T}^{2 m-1,1}(x, \xi) .
\end{aligned}
$$

Setting $\xi=(\lambda \zeta)^{1 / m} \varsigma, \zeta \in[0,+\infty), \varsigma \in \mathbb{S}^{n-1}, \lambda \geq \lambda_{0}$, we can rewrite $I_{2}(\lambda)$ as

$$
\begin{aligned}
& I_{2}(\lambda) \\
& =\frac{n}{m} \frac{\lambda^{n / m}}{(2 \pi)^{n}} \\
& \times \int e^{i \lambda\left(-t+\zeta t q_{\psi}(x, \zeta)+t \lambda^{-1} S^{m-1,1}\left(x,(\lambda \zeta)^{1 / m} \varsigma\right)+t^{2} \lambda^{-1} S_{T}^{2 m-1,1}\left(x,(\lambda \zeta)^{1 / m} \varsigma\right)\right)} \\
& \quad \times \psi(t) a\left(t ; x,(\lambda \zeta)^{1 / m} \varsigma\right) \\
& \quad \times H_{1}\left(\frac{\langle x\rangle\left\langle(\lambda \zeta)^{1 / m} \varsigma\right\rangle^{m}}{\lambda}\right) \\
& \quad \times\left[1-H_{2}\left((\lambda \zeta)^{1 / m}\right)\right] \zeta^{(n / m)-1} d t d \zeta d \varsigma d x \\
& =\frac{n}{m} \frac{\lambda^{n / m}}{(2 \pi)^{n}} \int e^{i \lambda F_{2}(X, Y ; \lambda)} U_{2}(X, Y ; \lambda) d X d Y,
\end{aligned}
$$

$X=(t, \zeta), Y=(\varsigma, x)$, where we have set

$$
\begin{aligned}
F_{2}(X, Y ; \lambda)= & -t+\zeta t q_{\psi}(x, \varsigma)+t \lambda^{-1} S^{m-1,1}\left(x,(\lambda \zeta)^{1 / m} \varsigma\right) \\
& +t^{2} \lambda^{-1} S_{T}^{2 m-1,1}\left(x,(\lambda \zeta)^{1 / m} \varsigma\right) \\
U_{2}(X, Y ; \lambda)= & \psi(t) H_{1}\left(\frac{\langle x\rangle\left\langle(\lambda \zeta)^{1 / m} \varsigma\right\rangle^{m}}{\lambda}\right) \\
& \times\left[1-H_{2}\left((\lambda \zeta)^{1 / m}\right)\right] \\
& \times a\left(t ; x,(\lambda \zeta)^{1 / m} \varsigma\right) \zeta^{(n / m)-1} .
\end{aligned}
$$

On the support of $U_{2}$, we have

$$
\begin{gathered}
\frac{\langle x\rangle\left\langle(\lambda \zeta)^{1 / m} \varsigma\right\rangle^{m}}{\lambda} \sim 1, \\
(\lambda \zeta)^{1 / m}>1 \Longrightarrow\left\langle(\lambda \zeta)^{1 / m} \varsigma\right\rangle^{m}=\left\langle(\lambda \zeta)^{1 / m}\right\rangle^{m} \sim \lambda \zeta,
\end{gathered}
$$

so that

$$
\begin{gathered}
\frac{\langle x\rangle \lambda \zeta}{\lambda} \sim 1 \Longleftrightarrow \zeta \sim\langle x\rangle^{-1}, \\
|x|<\langle x\rangle \leq 2 k_{1}\left(k_{2}\right)^{-m} \lambda=\tilde{\varkappa} \lambda .
\end{gathered}
$$

For any fixed $Y \in \mathbb{S}^{n-1} \times \mathbb{R}^{n}$, we then have $X$ belonging to a compact set, uniformly with respect to $\lambda \geq \lambda_{0}$, say supp $\psi \times$ $\left[c^{-1}\langle x\rangle^{-1}, c\langle x\rangle^{-1}\right]$, for a suitable $c>1$.

Remark 26. Incidentally, we observe that a rough estimate of $\lambda^{n / m} I_{2}(\lambda)$ is

$$
\begin{aligned}
& \int e^{i \lambda F_{2}(X, Y ; \lambda)} U_{2}(X, Y ; \lambda) d X \\
& \prec\langle x\rangle^{-(n / m)+1} \int_{c^{-1}\langle x\rangle^{-1}}^{c\langle x\rangle^{-1}} d \zeta\left\langle\langle x\rangle^{-n / m}\right. \\
& \Longrightarrow \lambda^{n / m} \int e^{i \lambda F_{2}(X, Y ; \lambda)} U_{2}(X, Y ; \lambda) d X d Y \prec \lambda^{n}, \\
& \lambda \longrightarrow+\infty .
\end{aligned}
$$

An even less precise result would be the bound $\lambda^{n / m}$, using the convergence of the integral with respect to $x$ in the whole $\mathbb{R}^{n}$, given by $-(n / m)+n<0$.

The next lemma is immediate, and we omit the proof.

Lemma 27. $S_{T}^{s, \sigma}\left(x,(\lambda \zeta)^{1 / m} \varsigma\right)=S_{T}^{s, \sigma}\left(x,(\lambda \zeta)^{1 / m}\right)$ for any $\zeta \epsilon$ $[0,+\infty), x \in \mathbb{R}^{n}, \varsigma \in \mathbb{S}^{n-1}, \lambda \geq \lambda_{0}, m \in(0,1)$, and, for all $\gamma \in \mathbb{Z}_{+}^{2}$

$$
D_{X}^{\gamma} S_{T}^{s, \sigma}\left(x,(\lambda \zeta)^{1 / m}\right)=\zeta^{-\gamma_{2}} S_{T}^{s, \sigma}\left(x,(\lambda \zeta)^{1 / m}\right) .
$$

The main result of this section is as follows.

Proposition 28. If $k_{1}, k_{2}, \lambda_{0}>1$ are chosen large enough, one has

$$
I_{2}(\lambda)=\frac{n}{m} d_{0} \lambda^{(n / m)-1}+O\left(\lambda^{n-1}\right)+O\left(\lambda^{(n / m)-2}\right) .
$$

Explicitly,

$$
d_{0}=\frac{1}{(2 \pi)^{n-1}} \int_{\mathbb{R}^{n}} \int_{\mathbb{S}^{n-1}} \frac{1}{q_{\psi}(x, \varsigma)^{n / m}} d \varsigma d x
$$

We will prove Proposition 28 through various intermediate steps. First of all, arguing as in the proof of (58), exchanging the role of $x$ and $\xi$, we note that, for all $x \in \mathbb{R}^{n}$, $\varsigma \in \mathbb{S}^{n-1}$,

$$
A^{-1}\langle x\rangle \leq q_{\psi}(x, \varsigma) \leq A\langle x\rangle,
$$

$(x, \varsigma) \in \mathbb{R}^{n} \times \mathbb{S}^{n-1}$. We now study 


$$
\begin{aligned}
F_{2, X}^{\prime}(X, Y ; \lambda) & =\left(\begin{array}{c}
\partial_{t} F_{2}(X, Y ; \lambda) \\
\partial_{\zeta} F_{2}(X, Y ; \lambda)
\end{array}\right) \\
& =\left(\begin{array}{c}
-1+\frac{\zeta}{\zeta_{0}}+\lambda^{-1} S^{m-1,1}\left(x,(\lambda \zeta)^{1 / m}\right)+t \lambda^{-1} S_{T}^{2 m-1,1}\left(x,(\lambda \zeta)^{1 / m}\right) \\
t\left(q_{\psi}(x, \varsigma)+\lambda^{-1} \zeta^{-1} S^{m-1,1}\left(x,(\lambda \zeta)^{1 / m}\right)+t \lambda^{-1} \zeta^{-1} S_{T}^{2 m-1,1}\left(x,(\lambda \zeta)^{1 / m}\right)\right)
\end{array}\right),
\end{aligned}
$$

$X=(t, \zeta) \in S_{X}=\operatorname{supp} \psi \times\left[c^{-1}\langle x\rangle^{-1}, c\langle x\rangle^{-1}\right], Y=$ $(\varsigma, x) \in S_{Y}=\mathbb{S}^{n-1} \times \mathbb{R}^{n}, \lambda \geq \lambda_{0}$, where we have used Lemma 27. By the symbolic calculus, remembering that $\lambda \zeta \geq$ $k_{2}^{m}>1$ on supp $U_{2}$, we can rewrite the expressions mentioned previously as

$$
\begin{aligned}
\partial_{t} F_{2}(X, Y ; \lambda)= & -1+\frac{\zeta}{\zeta_{0}}+\zeta(\lambda \zeta)^{-1} S^{m-1,1}\left(x,(\lambda \zeta)^{1 / m}\right) \\
& +t \zeta(\lambda \zeta)^{-1} S_{T}^{2 m-1,1}\left(x,(\lambda \zeta)^{1 / m}\right) \\
= & -1+\frac{\zeta}{\zeta_{0}}+\zeta\left[(\lambda \zeta)^{1 / m}\right]^{-m} S^{m-1,1}\left(x,(\lambda \zeta)^{1 / m}\right) \\
& +t \zeta\left[(\lambda \zeta)^{1 / m}\right]^{-m} S_{T}^{2 m-1,1}\left(x,(\lambda \zeta)^{1 / m}\right) \\
= & -1+\frac{\zeta}{\zeta_{0}}+\zeta S^{-1,1}\left(x,(\lambda \zeta)^{1 / m}\right) \\
& +t \zeta S_{T}^{m-1,1}\left(x,(\lambda \zeta)^{1 / m}\right), \\
\partial_{\zeta} F_{2}(X, Y ; \lambda)= & t\left(q_{\psi}(x, \zeta)+S^{-1,1}\left(x,(\lambda \zeta)^{1 / m}\right)\right. \\
& \left.+t S_{T}^{m-1,1}\left(x,(\lambda \zeta)^{1 / m}\right)\right) .
\end{aligned}
$$

It is clear that $\zeta \sim\langle x\rangle^{-1}$ implies $\zeta S^{-1,1}\left(x,(\lambda \zeta)^{1 / m}\right)=S^{-1,0}$ $\left(x,(\lambda \zeta)^{1 / m}\right)$ and $\zeta S_{T}^{m-1,1}\left(x,(\lambda \zeta)^{1 / m}\right)=S_{T}^{m-1,0}\left(x,(\lambda \zeta)^{1 / m}\right)$, so that we finally have

$$
\begin{aligned}
\partial_{t} F_{2}(X, Y ; \lambda)= & -1+\frac{\zeta}{\zeta_{0}}+S^{-1,0}\left(x,(\lambda \zeta)^{1 / m}\right) \\
& +t S_{T}^{m-1,0}\left(x,(\lambda \zeta)^{1 / m}\right), \\
\partial_{\zeta} F_{2}(X, Y ; \lambda)= & t\left(q_{\psi}(x, \varsigma)+S^{-1,1}\left(x,(\lambda \zeta)^{1 / m}\right)\right. \\
& \left.+t S_{T}^{m-1,1}\left(x,(\lambda \zeta)^{1 / m}\right)\right) .
\end{aligned}
$$

We now prove that, modulo an $O\left(|\lambda|^{-\infty}\right)$ term, we can consider an amplitude such that, on its support, the ration $\zeta / \zeta_{0}$ is very close to 1 . To this aim, take $H_{3} \in C_{0}^{\infty}(\mathbb{R})$ such that $0 \leq H_{3}(v) \leq 1, H_{3}(v)=1$ for $|v| \leq(3 / 2) \varepsilon$ and $H_{3}(v)=0$ for $|v| \geq 2 \varepsilon$, with an arbitrarily fixed, small enough $\varepsilon \in(0,1 / 2)$, and set

$$
\begin{aligned}
& V_{1}(X, Y ; \lambda)=U_{2}(X, Y ; \lambda) \cdot\left[1-H_{3}\left(\frac{\zeta}{\zeta_{0}}-1\right)\right], \\
& V_{2}(X, Y ; \lambda)=U_{2}(X, Y ; \lambda) \cdot H_{3}\left(\frac{\zeta}{\zeta_{0}}-1\right), \\
& J_{1}(\lambda)=\int e^{i \lambda F_{2}(X, Y ; \lambda)} V_{1}(X, Y ; \lambda) d X d Y, \\
& J_{2}(\lambda)=\int e^{i \lambda F_{2}(X, Y ; \lambda)} V_{2}(X, Y ; \lambda) d X d Y .
\end{aligned}
$$

Proposition 29. With the choices of $T, k_{1}, \lambda_{0}$, for any $\varepsilon \in$ $(0,1 / 2)$, one can find $k_{2}>1$ large enough such that $J_{1}(\lambda)=$ $O\left(\lambda^{-\infty}\right)$.

Proof. Since $0<m<1$, in view of (3), (74), and (79), we can choose $k_{2}>1$ so large that, for an arbitrarily fixed $\varepsilon \in(0,1 / 2)$, for any $\lambda \geq \lambda_{0}, \zeta \in(0,+\infty)$ satisfying $|\xi|=(\lambda \zeta)^{1 / m} \geq k_{2}$,

in $\partial_{t} F_{2}(X, Y ; \lambda)$,

$$
\begin{aligned}
& \left|S^{-1,0}\left(x,(\lambda \zeta)^{1 / m}\right)\right| \leq \frac{\varepsilon}{2} \\
& \left|t S_{T}^{m-1,0}\left(x,(\lambda \zeta)^{1 / m}\right)\right| \leq \frac{\varepsilon}{2}, \\
& \left.\left|\zeta_{0} \frac{d}{d \zeta} S^{-1,0}\left(x,(\lambda \zeta)^{1 / m}\right)\right|=\mid \zeta_{0} \zeta^{-1} S^{-1,0}\left(x,(\lambda \zeta)^{1 / m}\right)\right) \mid \\
& \leq k_{0}<1 \\
& \left|S^{-1,1}\left(x,(\lambda \zeta)^{1 / m}\right)+t S_{T}^{m-1,1}\left(x,(\lambda \zeta)^{1 / m}\right)\right| \\
& \leq \frac{A^{-1}}{2}\langle x\rangle
\end{aligned}
$$


uniformly with respect to $(X, Y) \in S_{X} \times S_{Y} \supseteq \operatorname{supp} U_{2}(\cdot ; \lambda)$. Then, $F_{2}$ is nonstationary on $\operatorname{supp} V_{1}$, since there we have $\left|\left(\zeta / \zeta_{0}\right)-1\right| \geq(3 / 2) \varepsilon$, while

$$
\left|S^{-1,0}\left(x,(\lambda \zeta)^{1 / m}\right)+t S_{T}^{m-1,0}\left(x,(\lambda \zeta)^{1 / m}\right)\right| \leq \varepsilon,
$$

which implies $\partial_{t} F_{2}(X, Y ; \lambda)>1$. Observing that, on supp $V_{1}$, $\partial_{t} F_{2}(X, Y ; \lambda)=S_{T}^{0,0}\left(x,(\lambda \zeta)^{1 / m}\right)$, as well as $V_{1}(X, Y ; \lambda)=$ $S_{T}^{0,0}\left(x,(\lambda \zeta)^{1 / m}\right)$, the assertion follows by repeated integrations by parts with respect to $t$, using the operator

$$
\begin{aligned}
L_{t} & =\frac{1}{\lambda \partial_{t} F_{2}(X, Y ; \lambda)} D_{t} \Longrightarrow L_{1} e^{i \lambda F_{2}(X, Y ; \lambda)} \\
& =e^{i \lambda F_{2}(X, Y ; \lambda)},
\end{aligned}
$$

and recalling Remark 26.

Proposition 30. With the choices of $\varepsilon, T>0, k_{1}, k_{2}, \lambda_{0}>1$, one can assume, modulo an $\mathrm{O}\left(\lambda^{n-1}\right)$ term, that the integral with respect to $x$ in $J_{2}(\lambda)$ is extended to the set $\left\{x \in \mathbb{R}^{n}:\langle x\rangle \leq \varkappa \lambda\right\}$, with

$$
\varkappa=\left(1-\frac{\varepsilon}{2}\right)\left[A\left(2 k_{2}\right)^{m}\right]^{-1}
$$

Proof. Indeed if $\varkappa<\tilde{\varkappa}=2 k_{1}\left\langle k_{2}\right\rangle^{-m}$, we can split $J_{2}(\lambda)$ into the sum

$$
\begin{aligned}
& \int_{\mathcal{\varkappa} \lambda \leq\langle x\rangle \leq \tilde{\mathcal{u}} \lambda} \int_{\mathbb{S}^{n-1}} \int e^{i \lambda F_{2}} V_{2} d X d \varsigma d x \\
& +\int_{\langle x\rangle \leq \mathcal{}} \int_{\mathbb{S}^{n-1}} \int e^{i \lambda F_{2}} V_{2} d X d \varsigma d x,
\end{aligned}
$$

since the inequality $\varkappa<\tilde{\varkappa}$ is true when $k_{2}$ is sufficiently large.

Observing that, on $\operatorname{supp} U_{2}$,

$$
\langle x\rangle \sim \lambda \Longrightarrow\langle\xi\rangle^{m}=\frac{\langle x\rangle\langle\xi\rangle^{m}}{\lambda} \frac{\lambda}{\langle x\rangle} \sim 1 \Longrightarrow|\xi| \leq k_{3},
$$

switching back to the original variables, the first integral in (88) can be treated as $I_{1}(\lambda)$, and gives, in view of Proposition 25, an $O\left(\lambda^{n-1}\right)$ term, as stated.

Now we can show that $F_{2}(X, Y ; \lambda)$ admits a unique, nondegenerate stationary point $X_{0}^{*}=X_{0}^{*}(Y, \lambda)$ belonging to $\operatorname{supp} V_{2}$ for $\langle x\rangle \leq \varkappa \lambda$. Under the same hypotheses, $X_{0}^{*}$ lies in a circular neighbourhood of $X_{0}=\left(0, \zeta_{0}\right)=\left(0, q_{\psi}(x, \varsigma)^{-1}\right)$ of arbitrarily small radius.

Proposition 31. With $\varepsilon \in(0,1 / 2), T>0, k_{1}, k_{2}, \lambda_{0}>1$ fixed previously, $F_{2, X}^{\prime}(X, Y ; \lambda)$ vanishes on supp $V_{2}$ only for $X=$
$X_{0}^{*}(Y ; \lambda)=\left(0, \zeta_{0}^{*}(Y ; \lambda)\right)$, that is, $F_{2, X}^{\prime}\left(X_{0}^{*}(Y ; \lambda), Y ; \lambda\right)=0$ for all $Y$ such that $(X, Y ; \lambda) \in \operatorname{supp} V_{2}$. Moreover,

$$
\begin{aligned}
& \operatorname{det}\left(F_{2, X}^{\prime \prime}\left(X_{0}^{*}(Y ; \lambda), Y\right)\right) \sim\langle x\rangle^{2}, \\
&\left|X_{0}^{*}(Y ; \lambda)-X_{0}(Y)\right|=\left|\zeta_{0}^{*}(Y ; \lambda)-\zeta_{0}(Y)\right| \\
& \leq \frac{A \varepsilon}{2}\langle x\rangle^{-1}
\end{aligned}
$$

holds on $\operatorname{supp} V_{2}$.

Proof. We have to solve

$$
\begin{gathered}
0=-1+\frac{\zeta}{\zeta_{0}}+S^{-1,0}\left(x,(\lambda \zeta)^{1 / m}\right) \\
+t S_{T}^{m-1,0}\left(x,(\lambda \zeta)^{1 / m}\right) \\
0=t\left(q_{\psi}(x, \varsigma)+S^{-1,1}\left(x,(\lambda \zeta)^{1 / m}\right)\right. \\
\left.+t S_{T}^{m-1,1}\left(x,(\lambda \zeta)^{1 / m}\right)\right),
\end{gathered}
$$

$(X, Y ; \lambda) \in \operatorname{supp} V_{2}$. By (79) and (84a) and (84b), with the choices of $\varepsilon, T>0, k_{1}, k_{2}, \lambda_{0}$, the coefficient of $t$ in the second equation does not vanish at any point of $\operatorname{supp} V_{2}$. Then $t=0$, and $\zeta$ must satisfy

$$
\begin{aligned}
-1+\frac{\zeta}{\zeta_{0}} & +S^{-1,0}\left(x,(\lambda \zeta)^{1 / m}\right)=0 \\
& \Longleftrightarrow \zeta=\zeta_{0}\left(1+S^{-1,0}\left(x,(\lambda \zeta)^{1 / m}\right)\right) \\
& =G(\zeta ; Y ; \lambda) .
\end{aligned}
$$

Since, by the choice of $k_{2},\left|\partial_{\zeta} G(\zeta ; Y ; \lambda)\right| \leq k_{0}<1$, uniformly with respect to $Y \in \mathbb{S}^{n-1} \times\left\{x \in \mathbb{R}^{n}:\langle x\rangle \leq \varkappa \lambda\right\}, \lambda \geq \lambda_{0}, G$ has a unique fixed point $\zeta_{0}^{*}=\zeta_{0}^{*}(Y ; \lambda)$, smoothly depending on the parameters; see the Appendix for more details. Since

$$
\begin{aligned}
& \partial_{t}^{2} F_{2}(X, Y ; \lambda)=S_{T}^{m-1,0}\left(x,(\lambda \zeta)^{1 / m}\right), \\
& \partial_{t} \partial_{\zeta} F_{2}(X, Y ; \lambda) \\
& =q_{\psi}(x, \varsigma)\left(1+\zeta_{0} \zeta^{-1}\left(S^{-1,0}\left(x,(\lambda \zeta)^{1 / m}\right)\right.\right. \\
& \left.\left.+t S_{T}^{m-1,0}\left(x,(\lambda \zeta)^{1 / m}\right)\right)\right), \\
& \partial_{\zeta}^{2} F_{2}(X, Y ; \lambda)=t \zeta^{-1}\left(S^{-1,1}\left(x,(\lambda \zeta)^{1 / m}\right)\right. \\
& \left.+t S_{T}^{m-1,1}\left(x,(\lambda \zeta)^{1 / m}\right)\right),
\end{aligned}
$$

we can assume that $\lambda \zeta \geq k_{2}^{m}$ and the choices of the other parameters imply, on $\operatorname{supp} V_{2}$,

$$
\begin{gathered}
\partial_{t}^{2} F_{2}(X, Y ; \lambda) \prec \frac{\varepsilon}{2}, \quad \partial_{t} \partial_{\zeta} F_{2}(X, Y ; \lambda) \sim\langle x\rangle, \\
\partial_{\zeta}^{2} F_{2}(X, Y ; \lambda) \prec \frac{\varepsilon}{2}\langle x\rangle^{2} .
\end{gathered}
$$

So we have proved that, on supp $V_{2}$, 


$$
\begin{aligned}
M & =F_{2, X}^{\prime \prime}\left(X_{0}^{*}(Y ; \lambda), Y ; \lambda\right)=\left(\begin{array}{cc}
M_{11} & M_{12} \\
M_{12} & 0
\end{array}\right) \\
& =\left(\begin{array}{c}
S_{T}^{m-1,0}\left(x,\left(\lambda \zeta_{0}^{*}\right)^{1 / m}\right) \\
q_{\psi}(x, \varsigma)\left[1+\frac{\zeta_{0}}{\zeta_{0}^{*}} S^{-1,0}\left(x,\left(\lambda \zeta_{0}^{*}\right)^{1 / m}\right)\right]
\end{array}\right) \\
& \Longrightarrow \operatorname{det}(M)=-q_{\psi}(x, \varsigma)^{2}\left[1+\frac{\zeta_{0}}{\zeta_{0}^{*}(Y ; \lambda)} S^{-1,0}\left(x,\left(\lambda \zeta_{0}^{*}(Y ; \lambda)\right)^{1 / m}\right)\right]^{2} \sim\langle x\rangle^{2}, \quad\|M\| \sim\langle x\rangle .
\end{aligned}
$$

By (3), (92), and $\zeta_{0}^{*}=G\left(\zeta_{0}^{*} ; Y ; \lambda\right),(X, Y) \in S_{X} \times S_{Y} \supseteq$ $\operatorname{supp} V_{2}(\cdot ; \lambda)$, we also find

$$
\begin{aligned}
\left|X_{0}^{*}(Y ; \lambda)-X_{0}(Y)\right| & =\left|\zeta_{0}^{*}(Y ; \lambda)-\zeta_{0}(Y)\right| \\
& =\left|\zeta_{0} S^{-1,0}\left(x,\left(\lambda \zeta_{0}^{*}(Y ; \lambda)\right)^{1 / m}\right)\right| \\
& \leq \frac{A \varepsilon}{2}\langle x\rangle^{-1},
\end{aligned}
$$

uniformly with respect to $\lambda \geq \lambda_{0}$. The proof is complete.

Remark 32. The choice of $k_{2}$ depends only on the properties of $q$ and on the values of $k_{1}$ and $\varepsilon$; that is, we first fix $k_{1}>$ $4 A C>2 A C>2$ and $\varepsilon \in(0,1 / 2)$, then $T>0$ small enough as explained at the beginning of the proof of Proposition 25, then $k_{2}>1$ as explained in the proofs of Propositions 29 and 31 , then, finally, $\lambda_{0}>2 k_{1}\left\langle 2 k_{2}\right\rangle^{m}$.

The next lemma says that the presence in the amplitude of factors which vanish at $X=X_{0}^{*}$ implies the gain of negative powers of $\lambda$.

Lemma 33. Assume $\alpha \in \mathbb{Z}_{+}^{2},|\alpha|>0$,

$$
\begin{aligned}
W=W(X, Y ; \lambda) \prec & V_{2}(X, Y ; \lambda) t^{\alpha_{1}} \\
& \times\left[W_{\alpha_{1}+\alpha_{2}}(X, Y ; \lambda)\left(\zeta-\zeta_{0}^{*}(Y ; \lambda)\right)^{\alpha_{1}+\alpha_{2}}\right] \\
& \text { or } \\
W=W(X, Y ; \lambda) \prec & V_{2}(X, Y ; \lambda) t^{\alpha_{1}+\alpha_{2}} \\
& \times\left[W_{\alpha_{2}}(X, Y ; \lambda)\left(\zeta-\zeta_{0}^{*}(Y ; \lambda)\right)^{\alpha_{2}}\right],
\end{aligned}
$$

$W$ is smooth, $W_{k}(X, Y ; \lambda) \prec\langle x\rangle^{k}, k \in \mathbb{Z}_{+}$, and has a SGbehaviour as the factors appearing in the expression of $V_{2}$. Then

$$
\begin{aligned}
\int e^{i \lambda F_{2}(X, Y ; \lambda)} W(X, Y ; \lambda) d X \\
=\lambda^{-|\alpha|} \int e^{i \lambda F_{2}(X, Y ; \lambda)} \widetilde{W}(X, Y ; \lambda) d X,
\end{aligned}
$$

where $\widetilde{W}$ has the same SG-behaviour, support and $x$-order of $V_{2}$, including the powers of $\zeta$.
Proof. By arguments similar to those used in the proof of Proposition 29, on supp $W$

$$
\begin{gathered}
\partial_{\zeta} F_{2}(X, Y ; \lambda) \succ\langle x\rangle|t|, \\
\partial_{t} F_{2}(X, Y ; \lambda) \succ\langle x\rangle\left|\zeta-\zeta_{0}^{*}(Y ; \lambda)\right| .
\end{gathered}
$$

Assume that the first condition in (97) holds. Under the hypotheses, if $\alpha_{1}>0$, we can first insert $e^{i \lambda F_{2}(X, Y ; \lambda)}=$ $L_{\zeta}^{\alpha_{1}} e^{i \lambda F_{2}(X, Y ; \lambda)}$ in the left-hand side of (98), where $L_{\zeta}=$ $D_{\zeta} / \lambda \partial_{\zeta} F_{2}(X, Y ; \lambda)$, and integrate by parts $\alpha_{1}$ times. Similarly, if $\alpha_{2}>0$, we subsequently use $e^{i \lambda F_{2}(X, Y ; \lambda)}=L_{t}^{\alpha_{2}} e^{i \lambda F_{2}(X, Y ; \lambda)}$, $L_{t}=D_{t} / \lambda \partial_{t} F_{2}(X, Y ; \lambda)$, and integrate by parts $\alpha_{2}$ times. The assertion then follows, remembering that $\zeta$-derivatives of $W$ produce either an additional $\zeta^{-1}$ factor or a lowering of the exponent of $\zeta-\zeta_{0}^{*}$, and that $\zeta, \zeta_{0}^{*} \sim\langle x\rangle^{-1}$ on supp $W$. The proof in the case that the second condition in (97) holds is the same, using first $L_{\zeta}$ and then $L_{t}$.

Proof of Proposition 28. Define,

$$
\mathbb{Q}=\mathscr{Q}(X, Y ; \lambda)=\left\langle M\left(X-X_{0}^{*}(Y ; \lambda)\right), X-X_{0}^{*}(Y ; \lambda)\right\rangle,
$$

and, for $s \in[0,1]$,

$$
\begin{gathered}
\mathscr{F}_{s}(X, Y ; \lambda)=\mathscr{Q}(X, Y ; \lambda)+s \mathscr{G}(X, Y ; \lambda), \\
\mathscr{G}(X, Y ; \lambda)=F_{2}(X, Y ; \lambda)-\mathscr{Q}(X, Y ; \lambda) .
\end{gathered}
$$

Remembering that $F_{2}\left(X_{0}^{*}(Y), Y ; \lambda\right)=0, F_{2, X}^{\prime}\left(X_{0}^{*}(Y), Y ; \lambda\right)=$ $0, Q$ is the Taylor polynomial of degree two of $F_{2}$ at $X=$ $X_{0}^{*}$, so that $\mathscr{G}$ vanishes of order 3 at $X=X_{0}^{*}$. Obviously, $\mathscr{F}_{0}(X, Y ; \lambda)=\mathbb{Q}(X, Y ; \lambda)$ and $\mathscr{F}_{1}(X, Y ; \lambda)=F_{2}(X, Y ; \lambda)$. Write

$$
\mathscr{J}_{\tau}(s)=\int e^{i \lambda \mathscr{F}_{s}\left(X, Y ; \tau^{-1}\right)} V_{2}\left(X, Y ; \tau^{-1}\right) d X,
$$

$\tau \in\left(0, \lambda_{0}^{-1}\right]$, and consider the Taylor expansion of $\mathscr{J}_{\tau}(s)$ of order $2 \mathscr{N}-1, \mathcal{N}>1$, so that

$$
\left|\mathscr{F}_{\tau}(1)-\sum_{k=0}^{2 \cdot \mathcal{N}-1} \frac{\mathscr{F}_{\tau}^{(k)}(0)}{k !}\right| \leq \sup _{0<s<1} \frac{\left|\mathscr{F}_{\tau}^{(2 \mathcal{N})}(s)\right|}{(2 \mathcal{N}) !} .
$$


Since

$$
\begin{gathered}
\mathscr{J}_{\tau}^{(2 \mathscr{N})}(s)=(i \lambda)^{2 \mathcal{N}} \int e^{i \lambda \mathscr{F}_{s}\left(X, Y ; \tau^{-1}\right)} \mathscr{G}\left(X, Y ; \tau^{-1}\right)^{2 \mathscr{N}} \\
\times V_{2}\left(X, Y ; \tau^{-1}\right) d X .
\end{gathered}
$$

Remark 26 and Lemma 33 imply that $\left|\mathscr{J}_{\tau}^{(2 \mathscr{N})}(s)\right| \prec$ $\lambda^{-\mathcal{N}}\langle x\rangle^{-n / m}, \tau \in\left(0, \lambda_{0}^{-1}\right], s \in[0,1]$. Indeed, it is easy to see, by direct computation, that $\mathscr{G}$ can be bounded by linear combinations of expressions of the form

$$
\begin{gathered}
t^{3}, t^{2}\left[W_{1}(X, Y ; \tau)\left(\zeta-\zeta_{0}^{*}(Y ; \tau)\right)\right] \\
t\left[W_{2}(X, Y ; \tau)\left(\zeta-\zeta_{0}^{*}(Y ; \tau)\right)^{2}\right], \\
t\left[W_{3}(X, Y ; \lambda)\left(\zeta-\zeta_{0}^{*}(Y ; \tau)\right)^{3}\right],
\end{gathered}
$$

with $W_{k}, k \in \mathbb{Z}_{+}$, having the required properties. Then, the bound of $\mathscr{G}^{2 \mathcal{N}}$ will always contain a term of the type $t^{3 \mathcal{N}}\left[W_{3 \mathcal{N}}(X, Y ; \lambda)\left(\zeta-\zeta_{0}^{*}(Y ; \lambda)\right)^{3 \mathcal{N}}\right]$, which corresponds to the (minimum) value $|\alpha|=3 \mathscr{N}$ in (97).

Each term $\mathscr{J}_{\tau}^{(k)}(0), k=0, \ldots, 2 \mathcal{N}-1$, has the quadratic phase function $\mathbb{Q}$, which of course also satisfies

$$
\begin{gathered}
\partial_{\zeta} Q\left(X, Y ; \tau^{-1}\right)>\langle x\rangle|t| \\
\partial_{t} Q\left(X, Y ; \tau^{-1}\right)>\langle x\rangle\left|\zeta-\zeta_{0}^{*}\left(Y ; \tau^{-1}\right)\right| .
\end{gathered}
$$

Then, denoting by $\Gamma$ the Taylor expansion of $\mathscr{G}$ at $X_{0}^{*}$ of order $3 \mathcal{N}$, we observe that $\mathscr{G}^{k}-\Gamma^{k}$ can be bounded by polynomial expressions in $X-X_{0}^{*}$ of the kind appearing in the right-hand side of (97), with $|\alpha|=\mathcal{N}+k$ (cf. the proof of Theorem 7.7.5 in [31]). Setting

$$
\mathscr{T}_{\tau}^{k}=\int e^{i \lambda Q\left(X, Y ; \tau^{-1}\right)}\left(i \lambda \Gamma\left(X, Y ; \tau^{-1}\right)\right)^{k} V_{2}\left(X, Y ; \tau^{-1}\right) d X .
$$

Lemma 33 implies

$$
\mathscr{J}_{\tau}^{(k)}(0)-\mathscr{T}_{\tau}^{k} \prec \lambda^{-\mathcal{N}}\langle x\rangle^{-n / m}
$$

We now apply the stationary phase method to $\mathscr{T}_{\tau}^{k}$ and prove that

$$
\mathscr{J}_{\tau}(1) \sim \sum_{j=0}^{+\infty} d_{j}(Y ; \tau) \lambda^{-1-j}
$$

which is a consequence of

$$
\begin{gathered}
\mathscr{T}_{\tau}^{k} \sim \lambda^{-1} \operatorname{det}\left(\frac{M}{2 \pi i}\right)^{-1 / 2} \sum_{l} L_{l, k, Y, \tau} V_{2}, \\
L_{l, k, Y, \tau} V_{2}=\sum_{l}(2 i \lambda)^{-l}\left\langle M^{-1} D_{X}, D_{X}\right\rangle^{l}\left[(i \lambda \Gamma)^{k} V_{2}\right] \\
\times\left(X_{0}^{*}\left(Y ; \tau^{-1}\right), Y ; \tau^{-1}\right) / l !,
\end{gathered}
$$

with $M$ evaluated with $\tau^{-1}$ in place of $\lambda$. Recalling (95), it follows that the inverse matrix $M^{-1}$ satisfies, on $\operatorname{supp} V_{2}$,

$$
\begin{aligned}
& M^{-1}=\left(\begin{array}{cc}
0 & \frac{1}{M_{12}} \\
\frac{1}{M_{12}} & -\frac{M_{11}}{M_{12}^{2}}
\end{array}\right), \\
& \frac{1}{M_{12}} \prec\langle x\rangle^{-1}, \quad \frac{M_{11}}{M_{12}^{2}} \prec \varepsilon\langle x\rangle^{-2}, \quad\left\|M^{-1}\right\| \sim\langle x\rangle^{-1},
\end{aligned}
$$

in view of the ellipticity of the involved symbols. Then, the operators $L_{j, k, Y, \tau}, j, k \in \mathbb{Z}_{+}$, do not increase the $x$-order of the resulting function with respect to that of their arguments, $(i \lambda \Gamma)^{k} V_{2}$, which is the same of $V_{2}$, uniformly with respect to $\tau$. The proof of (110) then follows by Theorem 7.6.1, the proof of Lemma 7.7.3 and formula (7.6.7) in [31]; see also [26, 32]. Indeed, by the mentioned results,

$$
\begin{aligned}
& \mathscr{J}_{\tau}^{k}-\lambda^{-1} \operatorname{det}\left(\frac{M}{2 \pi i}\right)^{-1 / 2} \sum_{l \leq k+\mathcal{N}} L_{l, k, Y, \tau} V_{2} \\
& =\left[\mathscr{J}_{\tau}^{k}-\lambda^{-1} \operatorname{det}\left(\frac{M}{2 \pi i}\right)^{-1 / 2} \sum_{l \leq k+\mathcal{N}+1} L_{l, k, Y, \tau} V_{2}\right] \\
& +\lambda^{-1} \operatorname{det}\left(\frac{M}{2 \pi i}\right)^{-1 / 2} L_{k+\mathcal{N}+1, k, Y, \tau} V_{2} \\
& \prec \lambda^{-\mathcal{N}-3}\langle x\rangle^{-1} \sum_{|\beta| \leq 2} \| D_{X}^{\beta}\left\langle M^{-1} D_{X}, D_{X}\right\rangle^{k+\mathcal{N}+3} \\
& \times\left[\mathscr{G}^{k} V_{2}\right]\left(X, Y ; \tau^{-1}\right) \|_{L^{2}\left(\mathbb{R}_{X}^{2}\right)} \\
& +\lambda^{-\mathcal{N}-2}\langle x\rangle^{-1}\left|L_{k+\mathcal{N}+1, k, Y, \tau} V_{2}\left(X_{0}^{*}\left(Y ; \tau^{-1}\right), Y ; \tau^{-1}\right)\right| \\
& \prec \lambda^{-\mathcal{N}-3}\langle x\rangle^{-1}\left[\int_{c^{-1}\langle x\rangle^{-1}}^{c\langle x\rangle^{-1}} \zeta^{2((n / m)-3)} d \zeta\right]^{1 / 2} \\
& +\lambda^{-\mathcal{N}-2}\langle x\rangle^{-n / m} \\
& \prec \lambda^{-\mathcal{N}-3}\langle x\rangle^{(-n / m)+(3 / 2)}+\lambda^{-\mathcal{N}-2}\langle x\rangle^{-n / m} \\
& \prec \lambda^{-\mathcal{N}-1-(1 / 2)}\langle x\rangle^{-n / m}, \quad \lambda \longrightarrow+\infty,
\end{aligned}
$$

since $\langle x\rangle \prec \lambda$ on supp $V_{2}$. It is then enough to sum all the expansions of $\mathscr{J}_{\tau}^{k} / k !, k=0, \ldots, 2 \mathscr{N}-1$, and sort the terms by 
decreasing exponents of $\lambda$ (as in the proof of Theorem 7.7.5 in [31]) to obtain (109) with the usual expression

$$
\begin{aligned}
\widetilde{d}_{j}(Y ; \tau)= & \operatorname{det}\left(\frac{M}{2 \pi i}\right)^{-1 / 2} \\
& \times \sum_{k-l=j 2 k \geq 3 l} \sum i^{-j} 2^{-k}\left\langle M^{-1} D_{X}, D_{X}\right\rangle^{k} \\
& \times\left[(i \Gamma)^{l} V_{2}\right]\left(X_{0}^{*}\left(Y ; \tau^{-1}\right), Y ; \tau^{-1}\right),
\end{aligned}
$$

so that, in particular,

$$
\widetilde{d}_{j}(Y ; \tau) \prec\langle x\rangle^{-n / m}
$$

for any $j \in \mathbb{Z}_{+}, \tau \in\left(0, \lambda_{0}^{-1}\right]$. We can then integrate $\mathscr{J}_{\tau}(1)$ and its asymptotic expansions with respect to $Y \in \mathbb{S}^{n-1} \times\{x \in$ $\left.\mathbb{R}^{n}:\langle x\rangle \leq \varkappa \lambda\right\}$ and find

$$
\begin{aligned}
J_{2}(\lambda) & =\int_{\langle x\rangle \leq \varkappa \lambda} \int_{\mathbb{S}^{n-1}} \mathscr{J}_{\lambda^{-1}}(1) d Y \\
& \sim \sum_{j} \lambda^{-1-j} \int_{\langle x\rangle \leq \varkappa \lambda} \int_{\mathbb{S}^{n-1}} \widetilde{d}_{j}\left(Y ; \lambda^{-1}\right) d Y, \quad \lambda \longrightarrow+\infty
\end{aligned}
$$

Recall that $\psi(0)=1$ and $a(0, x, \xi)=1$, for all $x, \xi \in \mathbb{R}^{n}$. Moreover, for $\zeta=\zeta_{0}^{*}(Y ; \lambda)$, the factors $H_{1}, H_{2}$, and $H_{3}$ are identically equal to 1 (see the Appendix). Then, the coefficient of the leading term in (115) is given by

$$
\begin{aligned}
& \int \tilde{d}_{0}\left(Y ; \lambda^{-1}\right) d Y \\
&=\int_{\langle x\rangle \leq \varkappa \lambda} \int_{\mathbb{S}^{n-1}} \operatorname{det}\left(\frac{M}{2 \pi i}\right)^{-1 / 2} V_{2}\left(X_{0}^{*}(Y ; \lambda), Y ; \lambda\right) d Y \\
&=2 \pi \int_{\langle x\rangle \leq \varkappa \lambda} \int_{\mathbb{S}^{n-1}} H_{1}\left(\frac{\langle x\rangle\left\langle\left(\lambda \zeta_{0}^{*}(\varsigma, x ; \lambda)\right)^{1 / m}\right\rangle^{m}}{\lambda}\right) \\
& \quad \times H_{2}\left(\left(\lambda \zeta_{0}^{*}(\varsigma, x ; \lambda)\right)^{1 / m}\right) \\
& \times H_{3}\left(\frac{\zeta_{0}^{*}(\varsigma, x ; \lambda)}{\zeta_{0}(\varsigma, x)}-1\right) \\
& \times|\operatorname{det}(M)|^{-1 / 2} \zeta_{0}^{*}(\varsigma, x ; \lambda)^{(n / m)-1} d \varsigma d x \\
& \int_{\mathbb{S}^{n-1}}|\operatorname{det}(M)|^{-1 / 2} \zeta_{0}^{*}(\varsigma, x ; \lambda)^{(n / m)-1} d \varsigma d x,
\end{aligned}
$$

with $M$ evaluated in $\zeta=\zeta_{0}^{*}$. We say that

$$
\begin{aligned}
& \int \tilde{d}_{0}\left(Y ; \lambda^{-1}\right) d Y= 2 \pi \int_{\mathbb{R}^{n}} \int_{\mathbb{S}^{n-1}} \frac{1}{q_{\psi}(x, \varsigma)^{n / m}} d \varsigma d x \\
&+O\left(\lambda^{\max \{-1 / m, n-(n / m),-1\}}\right) \\
&= 2 \pi d_{0}+O\left(\lambda^{\max \{-1 / m, n-(n / m),-1\}}\right), \\
& \lambda \longrightarrow+\infty
\end{aligned}
$$

To confirm this, first note that $\zeta_{0}^{*}(Y ; \lambda) \rightarrow \zeta_{0}(Y), \lambda \rightarrow$ $+\infty$, for any $(Y ; \lambda)$ belonging to the support of the integrand, see the Appendix. Moreover, the integrand is uniformly bounded by the summable function $\langle x\rangle^{-n / m}$, and its support is included in the set $S$. Then, recalling (95) and setting $\widetilde{H}=$ $\left|\zeta_{0}^{2} \operatorname{det}(M)\right|^{-1 / 2}$,

$$
\begin{aligned}
R= & \int_{\langle x\rangle \leq \varkappa \lambda} \int_{\mathbb{S}^{n-1}}|\operatorname{det}(M)|^{-1 / 2} \zeta_{0}^{*}(Y ; \lambda)^{(n / m)-1} d Y \\
& -\int_{\mathbb{R}^{n}} \int_{\mathbb{S}^{n-1}} \zeta_{0}(Y)^{n / m} d Y \\
= & \int_{\langle x\rangle \leq \varkappa \lambda} \int_{\mathbb{S}^{n-1}} \zeta_{0}\left[\widetilde{H}\left(\zeta_{0}^{*}\right)^{(n / m)-1}-\zeta_{0}^{(n / m)-1}\right] d \varsigma d x \\
& -\int_{\langle x\rangle \geq \varkappa \lambda} \int_{\mathbb{S}^{n-1}} \zeta_{0}^{n / m} d \varsigma d x .
\end{aligned}
$$

The second integral is always $O\left(\lambda^{n-(n / m)}\right)$, since $q_{\psi}(x, \varsigma) \sim$ $\langle x\rangle$ implies

$$
\begin{aligned}
R_{2} & =\int_{\langle x\rangle \geq \varkappa \lambda} \int_{\mathbb{S}^{n-1}} \zeta_{0}^{n / m} d \varsigma d x \\
& \sim \int_{\varkappa \lambda}^{+\infty} r^{n-(n / m)-1} d r=\frac{(\varkappa \lambda)^{n-(n / m)}}{(n / m)-n}, \quad \lambda \longrightarrow+\infty .
\end{aligned}
$$

The first integral can be estimated as follows. Since

$$
\zeta_{0}^{*}-\zeta_{0}=\zeta_{0} S^{-1,0}\left(x,\left(\lambda \zeta_{0}^{*}\right)^{1 / m}\right)=\zeta_{0} O\left(\left(\lambda \zeta_{0}^{*}\right)^{-1 / m}\right),
$$

by the properties of $\zeta_{0}^{*}$ (see the appendix) we find

$$
\begin{aligned}
\left(\frac{\zeta_{0}^{*}}{\zeta_{0}}\right)^{(n / m)-1}-1 & =\left(1+O\left(\left(\lambda \zeta_{0}^{*}\right)^{-1 / m}\right)\right)^{(n / m)-1}-1 \\
& =O\left(\left(\lambda \zeta_{0}^{*}\right)^{-1 / m}\right)=O\left(\langle x\rangle^{1 / m} \lambda^{-1 / m}\right),
\end{aligned}
$$

since $S^{-1,0}\left(x,\left(\lambda \zeta_{0}^{*}\right)^{1 / m}\right) \ll 1$. By (95), we similarly have $\widetilde{H}=$ $1+O\left(\langle x\rangle^{1 / m} \lambda^{-1 / m}\right)$, so that

$$
\begin{aligned}
R_{1} & =\int_{\langle x\rangle \leq \varkappa \lambda} \int_{\mathbb{S}^{n-1}} \zeta_{0}\left[\widetilde{H}\left(\zeta_{0}^{*}\right)^{(n / m)-1}-\zeta_{0}^{(n / m)-1}\right] d \varsigma d x \\
& =\int_{\langle x\rangle \leq \varkappa \lambda} \int_{\mathbb{S}^{n-1}} \zeta_{0}^{n / m}\left[\widetilde{H}\left(\frac{\zeta_{0}^{*}}{\zeta_{0}}\right)^{(n / m)-1}-1\right] d \varsigma d x \\
& \prec \lambda^{-1 / m} \int_{\langle x\rangle \leq \varkappa \lambda}\langle x\rangle^{-(n-1) / m} d x .
\end{aligned}
$$


If $n>1 /(1-m) \Leftrightarrow n-1-((n-1) / m)<-1, n \in$ $\mathbb{N}, m \in(0,1)$, the integral in $R_{1}$ is convergent for $\lambda \rightarrow$ $+\infty$ and $R_{1}=O\left(\lambda^{-1 / m}\right)$. In this case, $R_{1}$ contributes an $O\left(\lambda^{(n / m)-1-(1 / m)}\right)$ term to the expansion of $I_{2}(\lambda)$, which is of lower order than the $O\left(\lambda^{(n / m)-2}\right)$ term, which is one of the remainders appearing in (77). On the other hand, if $n<$ $1 /(1-m)$, the integral in $R_{1}$ is divergent, and $R_{1}$ itself is $O\left(\lambda^{n-(n / m)}\right)$, since, trivially

$$
\begin{aligned}
& \lim _{\lambda \rightarrow+\infty} \frac{\lambda^{-1 / m} \int_{0}^{\varkappa \lambda}\left(r^{n-1} /\left(1+r^{2}\right)^{(n-1) / 2 m}\right) d r}{\lambda^{n-(n / m)}} \\
& =\lim _{\lambda \rightarrow+\infty} \frac{\int_{0}^{\varkappa \lambda}\left(r^{n-1} /\left(1+r^{2}\right)^{(n-1) / 2 m}\right) d r}{\lambda^{(n-((n-1) / m))}} \\
& =\frac{\varkappa^{n-1-((n-1) / m)}}{n-((n-1) / m)} .
\end{aligned}
$$

Finally, if $n=1 /(1-m), R_{1}$ is $O\left(\lambda^{-1 / m} \ln \lambda\right)$, by

$$
\lim _{\lambda \rightarrow+\infty} \frac{\int_{0}^{\varkappa \lambda}\left(r^{1 /(1-m)-1} /\left(1+r^{2}\right)^{1 / 2(1-m)}\right) d r}{\ln \lambda}=\varkappa^{-1},
$$

and again contributes a term of lower order than the remain$\operatorname{der} O\left(\lambda^{(n / m)-2}\right)$. Similar conclusions can be obtained for the subsequent terms of the expansion of $J_{2}(\lambda)$.

The proof is complete, combining the contributions of the remainders like $R$ with the other terms in the expansion of $J_{2}(\lambda)$, and remembering that

$$
\begin{aligned}
I_{2}(\lambda)= & \frac{n}{m} \frac{\lambda^{n / m}}{(2 \pi)^{n}} J_{2}(\lambda)+O\left(|\lambda|^{-\infty}\right) \\
= & \frac{n}{m} \lambda^{n / m} \sum_{j=0}^{+\infty}\left(d_{j} \lambda^{-1-j}+O\left(\lambda^{n-(n / m)-1-j}\right)\right) \\
& +O\left(|\lambda|^{-\infty}\right) .
\end{aligned}
$$

Remark 34. The same conclusions concerning the behaviour of $R_{1}$ in the final step of the proof of Proposition 28 could have been obtained studying the Taylor expansion of the extension of $\zeta_{0}^{*}\left(Y ; \tau^{-1}\right), \tau=\lambda^{-1}$, to the interval $\left[0, \lambda_{0}^{-1}\right]_{\tau}$, similarly to [32].

Proof of Theorem 1. The statement for $\mu>m$ follows by the arguments in Section 3 and Propositions 23, 25 and 28, summing up the contribution of the local symbol on the exit chart to the contributions of the remaining local symbols, which gives the desired multiple of the integral of $q_{\psi}^{-n / m}$ on the cosphere bundle as coefficient of the leading term $\lambda^{n / m}$. The remainder has then order equal to the maximum between $(n / m)-1$ and $n$, as claimed. The proof for $\mu<m$ is the same, by exchanging step by step the role of $x$ and $\xi$.

\section{Appendix}

For the sake of completeness, here we illustrate some details of the proof of Theorem 1, which we skipped in the previous sections. They concern, in particular, formula (41), which expresses the relation between $\sum_{j} \widehat{\psi}\left(\lambda-\eta_{j}\right)$ and the oscillatory integrals examined in Section 4 . We mainly focus on the aspects which are specific for the manifolds with ends.

We also show more precisely how the constants $k_{1}, k_{2}, \lambda$ are involved in the solution of (92) via the fixed point theorem, completing the proof of Proposition 31.

\section{A. Solution of Cauchy Problems and SG Fourier Integral Operators}

Using the so-called "geometric optics method", specialised to che pseudodifferential calculus we use (see $[22-24,28,29$, $33]$ ), the Cauchy problem (33) can be solved modulo $\mathcal{S}(M)$ by means of an operator family $V(t)$, defined for $t$ in a suitable interval $(-T, T), T>0: V(t)$ induces continuous maps

$$
\begin{gathered}
V: \mathcal{S}(M) \longrightarrow C^{\infty}((-T, T), \mathcal{S}(M)), \\
V: \mathcal{S}^{\prime}(M) \longrightarrow C^{\infty}\left((-T, T), \mathcal{S}^{\prime}(M)\right), \\
\left(D_{t}-Q\right) \circ V=: R \in C^{\infty}\left((-T, T), L^{-\infty,-\infty}(M)\right), \\
V u_{\mid t=0}=u, \quad \forall u \in \mathcal{S}^{\prime}(M) .
\end{gathered}
$$

First of all, we recall that the partition of unity $\left\{\theta_{k}\right\}$ and the family of functions $\left\{\chi_{k}\right\}$ of Definition 8 can be chosen so that $\left(\theta_{k}\right)_{*}$ and $\left(\chi_{k}\right)_{*}$ are SG-symbols of order $(0,0)$ on $U_{k}$, extendable to symbols of the same class defined on $\mathbb{R}^{n}$ (see [5]).

Remark A.1. (1) The complete symbol of $Q$ depends, in general, on the choice of the admissible atlas, of $\left\{\theta_{k}\right\}$ and of $\left\{\chi_{k}\right\}$. Anyway, if $\left\{\tilde{q}_{k}\right\}$ is another complete symbol of $Q$, $\kappa(x)\left(q_{k}(x, \xi)-\widetilde{q}_{k}(x, \xi)\right) \in \mathcal{S}\left(\varphi_{k}\left(\Omega_{k} \cap \widetilde{\Omega}_{k}\right)\right)$ for an admissible cutoff function $\kappa$ supported in $\varphi_{k}\left(\Omega_{k} \cap \widetilde{\Omega}_{k}\right)$.

(2) The solution of (33) in the SG-classical case and the properties of $\varphi_{k}$ and $a_{k}$ in (37) were investigated in [28] (see also [33, Section 4]). In particular, it turns out that $\varphi_{k} \in$ $C^{\infty}\left(\left(-T_{k}, T_{k}\right), S_{\mathrm{cl}}^{1,1}\right), T_{k}>0$. According to ([23, page 101]), for every SG phase functions $\varphi$ of the type involved in the definition of $V(t)$ we also have, for all $x \in \mathbb{R}^{n}$,

$$
\begin{aligned}
\left|\nabla_{\xi} \varphi(t ; x, \xi)-x\right| & =\left|\nabla_{\xi} \varphi(t ; x, \xi)-\nabla_{\xi} \varphi(0 ; x, \xi)\right| \\
& =\left|\int_{0}^{t} \nabla_{\xi} \dot{\varphi}(t ; x, \xi) d t\right| \\
& =\left|\int_{0}^{t} \nabla_{\xi}\left(q\left(x, d_{x} \varphi(t ; x, \xi)\right)\right) d t\right| \\
& \leq C|t|\langle x\rangle,
\end{aligned}
$$

with a constant $C>0$ not depending on $t, x, \xi$. The function $\Phi_{t, \xi}(x):=\nabla_{\xi} \varphi(t, x, \xi)$ turns out to be a (SG-) diffeomorphism, smoothly depending on the parameters $t$ and $\xi$ (see [22]). 
Before proving Theorem 16, we state a technical lemma, whose proof is immediate and henceforth omitted.

Lemma A.2. Let $U \subset \mathbb{R}^{n}$ be an open set and define $U_{\delta}:=$ $\bigcup_{x \in U} B(x, \delta\langle x\rangle)$ for arbitrary $\delta>0$. Assume $\theta, \chi \in C^{\infty}\left(\mathbb{R}^{n}\right)$ such that $\operatorname{supp} \theta \subset U_{\delta / 5}$, supp $\chi \subset U_{\delta}$ and $\left.\chi\right|_{U_{\delta / 2}} \equiv 1$. Then, for any diffeomorphism $\Phi_{t, \xi}$, smoothly depending on $t \in(-T, T)$, $\xi \in \mathbb{R}^{n}$, and such that for all $t, x, \xi\left|\Phi_{t, \xi}(x)-x\right| \leq C|t|\langle x\rangle$ with a constant $C>0$ independent of $t, x, \xi$,

$$
|t|<\frac{\delta}{4 C} \Longrightarrow(1-\chi(x))\left(\partial^{\alpha} \theta\right)\left(\Phi_{t, \xi}(x)\right)=0
$$

for any multi-index $\alpha$ and $x, \xi \in \mathbb{R}^{n}$.

We remark that, since a manifold with ends is, in particular, a SG-manifold, the charts $\left(\Omega_{k}, \psi_{k}\right)$, and the functions $\left\{\theta_{k}\right\}$, $\left\{\chi_{k}\right\}$ can be chosen such that

(i) for a fixed $\delta>0$, each coordinate open set $U_{k}=$ $\psi_{k}\left(\Omega_{k}\right), k=1, \ldots, N$, contains an open subset $W_{k}$ such that $\bigcup_{x \in W_{k}} B(x, \delta\langle x\rangle) \subseteq U_{k}$;

(ii) the supports of $\theta_{k}$ and $\chi_{k}, k=1, \ldots, N$, satisfy hypotheses as the supports of $\theta$ and $\chi$ in Lemma A.2 (see, e.g., Section 3 of [5] for the construction of functions with the required properties).

In fact, this is relevant only for $k=N$.

Proof of Theorem 16. We will write $R \equiv S$ when $R-$ $S \in L^{-\infty,-\infty}(M)$ and $\chi_{k} \triangleleft \tilde{\chi}_{k}$ when the functions $\chi_{k}, \tilde{\chi}_{k}$ are smooth, nonnegative, supported in $\Omega_{k}$, satisfy $\chi_{k} \widetilde{\chi}_{k}=\chi_{k}$ and $\left(\chi_{k}\right)_{*},\left(\tilde{\chi}_{k}\right)_{*}$ are SG-symbols of order $(0,0)$ on $U_{k}=\psi_{k}\left(\Omega_{k}\right)$. Obviously, $R \in L^{-\infty,-\infty}(M)$ implies $R V \in C^{\infty}\left((-T, T), L^{-\infty,-\infty}(M)\right)$. To simplify notation, in the following computations we will not distinguish between the functions $\chi_{k}, \theta_{k}$, and so forth, and their local representations.

$V(t)$ obviously satisfies (A.3). To prove (A.2), choose functions $\zeta_{k}, v_{k}$ supported in $\Omega_{k}$ such that $\theta_{k} \triangleleft \zeta_{k} \triangleleft \chi_{k} \triangleleft v_{k}$. Then $Q \equiv \sum_{k=1}^{N} \theta_{k} Q_{k} \chi_{k}$ and, for all $k=1, \ldots, N, Q \chi_{k} \equiv$ $v_{k} Q_{k} \chi_{k}$ (see [1], Section 4.4; cf. also [11]), so that

$$
\begin{aligned}
& Q V(t)= \sum_{k=1}^{N} Q \chi_{k} V_{k}(t) \theta_{k} \equiv \sum_{k=1}^{N} v_{k} Q_{k} \chi_{k} V_{k}(t) \theta_{k} \\
&= \sum_{k=1}^{N}\left(v_{k}\left[Q_{k}, \chi_{k}\right] V_{k}(t) \theta_{k}+\chi_{k} Q_{k} V_{k}(t) \theta_{k}\right) \\
& \equiv \sum_{k=1}^{N}\left(v_{k}\left[Q_{k}, \chi_{k}\right] \zeta_{k} V_{k}(t) \theta_{k}\right. \\
&\left.\quad+v_{k}\left[Q_{k}, \chi_{k}\right]\left(1-\zeta_{k}\right) V_{k}(t) \theta_{k}\right) \\
& \quad+D_{t} V(t) \\
& \equiv D_{t} V(t) .
\end{aligned}
$$

That the first term in the sum (A.6) is smoothing comes from the SG symbolic calculus in $\mathbb{R}^{n}$ and the observations above, since $\operatorname{sym}\left(\left[Q_{k}, \chi_{k}\right] \zeta_{k}\right) \sim 0$. The same property holds for each $k$ in the second term, provided $t \in I_{T_{k}}, T_{k}>0$ small enough. In fact, by Theorems 7 and 8 of [22], $\left(1-\zeta_{k}\right) V_{k}(t) \theta_{k}$ is a SG FIO with the same phase function $\varphi_{k}$ and amplitude $w_{k}$ such that

$$
w_{k}(x, \xi) \sim \sum_{\alpha} \frac{\left(1-\zeta_{k}(x)\right)\left(\partial^{\alpha} \theta_{k}\right)\left(\nabla_{\xi} \varphi_{k}(t ; \xi, x)\right)}{\alpha !} b_{j \alpha}(t ; x, \xi),
$$

with suitable SG-symbols $b_{j \alpha}$ defined in terms of $\varphi_{k}$ and $a_{k}$. By Remark A.1 and Lemma A.2, $w_{k} \sim 0$ for $|t|$ small enough. The proof that $V(t)$ satisfies (A.2) is completed once we set $T=$ $\min \left\{T_{1}, \ldots, T_{N}\right\}$. The last part of the theorem can be proved as in [25], Proposition 12.3, since, setting $W(t):=U(-t) V(t)$, it is easy to see $D_{t} W(t) \equiv 0$, so that $W(0)=I \Rightarrow W(t) \equiv I \Rightarrow$ $V(t) \equiv U(t)$, with smooth dependence on $t$, as claimed.

\section{B. Trace Formula and Asymptotics for}

$$
A \in E L_{\mathrm{cl}}^{r, 1}(M)
$$

Proof of Lemma 15. Consider first the finite sum

$$
k_{J}(x, y)=\sum_{j=1}^{J} \widehat{\psi}\left(-\eta_{k}\right) e_{k}(x) \overline{e_{k}(y)}
$$

and reduce to the local situation (cf. Schrohe [5]), via the SGcompatible partition of unity $\left\{\theta_{l}\right\}$ subordinate to the atlas $\mathscr{A}$, by

$$
\begin{aligned}
k_{J}(x, y) & =\sum_{r, s=1}^{N} \sum_{j=1}^{J} \widehat{\psi}\left(-\eta_{k}\right)\left(\theta_{r} e_{k}\right)(x) \overline{\left(e_{k} \theta_{s}\right)(y)} \\
& =\sum_{r, s=1}^{N} k_{R}^{r s}(x, y) .
\end{aligned}
$$

Then, by $e_{k} \in \mathcal{S}(M)$ and the fact that $\left(\theta_{r}\right)_{*}=\theta_{r} \circ$ $\psi_{r}^{-1}$ is supported and at most of polynomial growth in $U_{r}$, it turns out that we can extend $\left(\theta_{r} e_{k}\right)_{*}$ and $\left(\theta_{s} e_{k}\right)_{*}$ to elements of $\delta\left(\mathbb{R}^{n}\right)$. By an argument similar to the proof of Proposition 1.10.11 in [13] (or by direct estimates of the involved seminorms, as in [25]), $\left(k_{J}^{r s}\right)_{*} \rightarrow\left(k^{r s}\right)_{*}$ in $\delta\left(\mathbb{R}^{n} \times\right.$ $\mathbb{R}^{n}$ ) when $J \rightarrow+\infty$, with $\left(k^{r s}\right)_{*}$ kernel of $\left(\theta_{r} \widehat{\psi}(-Q) \theta_{s}\right)_{*}$. This proves that $\widehat{\psi}(-Q)=\sum_{r, s=1}^{N} \theta_{r} \widehat{\psi}(-Q) \theta_{s}$ is an operator with kernel $K_{\psi}(x, y)=\sum_{r, s=1}^{N} k^{r s}(x, y) \in \mathcal{S}(M \times M)$.

The proof of Theorem 19 is essentially the one in [25], while the proof of Lemma B.1 comes from [13]. We include both of them here for convenience of the reader.

Proof of Theorem 19. Setting $G(\lambda)=\int_{-\infty}^{\lambda} \widehat{\psi}(\tau) d \tau$ and integrating $(42)$ in $(-\infty, \lambda)$, we obtain

$$
\begin{aligned}
& \int_{-\infty}^{\lambda} \int \widehat{\psi}(\tau-\eta) d N_{Q}(\eta) d \tau \\
& \quad=\int\left(\int_{-\infty}^{\lambda} \widehat{\psi}(\tau-\eta) d \tau\right) d N_{Q}(\eta)
\end{aligned}
$$




$$
\begin{aligned}
& =\int G(\lambda-\eta) d N_{Q}(\eta) \\
& = \begin{cases}d_{0} \lambda^{n / m}+O\left(\lambda^{n^{*}}\right) & \text { for } \lambda \longrightarrow+\infty \\
O\left(|\lambda|^{-\infty}\right) & \text { for } \lambda \longrightarrow-\infty .\end{cases}
\end{aligned}
$$

Now, observe that

$$
\begin{aligned}
\int G(\lambda-\eta) d N_{Q}(\eta) & =\sum_{j=1}^{\infty} G\left(\lambda-\eta_{j}\right) \\
& =\sum_{j=1}^{\infty} \int_{-\infty}^{\lambda-\eta_{j}} \widehat{\psi}(\tau) d \tau \\
& =\sum_{j=1}^{\infty} \int H\left(\lambda-\eta_{j}-\tau\right) \widehat{\psi}(\tau) d \tau,
\end{aligned}
$$

where $H(\tau)$ is the Heaviside function. Bringing the series under the integral sign, we can write

$$
\begin{aligned}
\int G(\lambda-\eta) d N_{Q}(\eta)= & \int \sum_{j=1}^{\infty} H\left(\lambda-\eta_{j}-\tau\right) \widehat{\psi}(\tau) d \tau \\
= & \int N_{Q}(\lambda-\tau) \widehat{\psi}(\tau) d \tau \\
= & N_{Q}(\lambda) \int \widehat{\psi}(\tau) d \tau \\
& +\int\left[N_{Q}(\lambda-\tau)-N_{Q}(\lambda)\right] \widehat{\psi}(\tau) d \tau \\
= & 2 \pi N_{Q}(\lambda)+R(\lambda),
\end{aligned}
$$

since $\int \widehat{\psi}(\tau) d \tau=2 \pi \psi(0)=2 \pi$. In view of the monotonicity of $N$ and next Lemma B.1 (cf. Lemma 4.2.8 of [13]), for $\lambda \geq 1$

$$
\begin{aligned}
\left|N_{Q}(\lambda-\tau)-N_{Q}(\lambda)\right| & \leq N_{Q}(\lambda+|\tau|)-N_{Q}(\lambda-|\tau|) \\
& =\int_{\lambda-|\tau|}^{\lambda+|\tau|} d N_{Q}(\eta)=\int_{|\lambda-\eta| \leq|\tau|} d N_{Q}(\eta) \\
& \leq C(1+|\tau|)^{n / m}(1+|\lambda|)^{(n / m)-1} \\
& \leq \widetilde{C}(1+|\tau|)^{n / m} \lambda^{(n / m)-1} .
\end{aligned}
$$

We can then conclude that $R(\lambda)=O\left(\lambda^{n / m-1}\right), \lambda \geq 1$, since $\widehat{\psi} \in \mathcal{S}$, and this, together with (B.4) and (B.6), completes the proof.

Lemma B.1. Under the hypotheses of Theorem 19, there exists a constant $C>0$ such that for any $K \geq 0$ and any $\lambda \in \mathbb{R}$

$$
\int_{|\lambda-\eta| \leq K} d N_{Q}(\eta) \leq C(1+K)^{n / m}(1+|\lambda|)^{(n / m)-1} .
$$

Proof. Let $h \in(0, \widehat{\psi}(0))$ and $\left[-K_{0}, K_{0}\right]$ such that $\widehat{\psi}(t) \geq h$ for all $t \in\left[-K_{0}, K_{0}\right]$. Then, trivially,

$$
\int_{|\lambda-\eta| \leq K_{0}} d N_{Q}(\eta) \leq h^{-1} \int \widehat{\psi}(\lambda-\eta) d N_{Q}(\eta) .
$$

Let us now prove that

$$
\int \widehat{\psi}(\lambda-\eta) d N_{\mathrm{Q}}(\eta) \leq C_{1}(1+|\lambda|)^{(n / m)-1} .
$$

Indeed, this is clear for $\lambda \geq \widetilde{C}>0$ and $\lambda \leq-\widetilde{C}, \widetilde{C}$ suitably large, in view of hypothesis (iii). For $\lambda \in[-\widetilde{C}, \widetilde{C}]$, choose a constant $C_{1}$ so large that $\max _{\lambda \in[-\widetilde{C}, \widetilde{C}]} \int \widehat{\psi}(\lambda-\eta) d N_{Q}(\eta) \leq$ $C_{1}(1+\widetilde{C})^{(n / m)-1}$. This shows that, for all $\lambda \in \mathbb{R}$,

$$
\int_{|\lambda-\eta| \leq K_{0}} d N_{Q}(\eta) \leq C_{2}(1+|\lambda|)^{(n / m)-1} .
$$

For arbitrary $K>0$ there exists $l \in \mathbb{N}$ such that $(l-1) K_{0} \leq$ $K<l K_{0}$. We write

$$
\begin{aligned}
\int_{|\lambda-\eta| \leq K} d N_{Q}(\eta) \leq & \int_{|\lambda-\eta| \leq l K_{0}} d N_{Q}(\eta) \\
& =\sum_{j=0}^{l-1} \int_{j K_{0} \leq|\lambda-\eta| \leq(j+1) K_{0}} d N_{Q}(\eta) \\
\leq & \sum_{j=0}^{l-1}\left[\int_{\left|\lambda+j K_{0}+K_{0} / 2-\eta\right| \leq K_{0} / 2} d N_{Q}(\eta)\right. \\
& \left.\quad+\int_{\left|\lambda-j K_{0}-K_{0} / 2-\eta\right| \leq K_{0} / 2} d N_{Q}(\eta)\right] .
\end{aligned}
$$

By (B.11), the last sum can be estimated by

$$
\begin{aligned}
& 2 C_{2} \sum_{j=0}^{l-1}\left(1+|\lambda|+\left(j+\frac{1}{2}\right) K_{0}\right)^{(n / m)-1} \\
& \leq 2 C_{2} l\left(1+|\lambda|+\frac{K_{0}}{2}+K\right)^{(n / m)-1} \\
& \leq 2 C_{2}\left(1+\frac{K}{K_{0}}\right)\left(1+\frac{K_{0}}{2}+K\right)^{(n / m)-1} \\
& \times\left(1+\frac{1}{1+\left(K_{0} / 2\right)+K}|\lambda|\right)^{(n / m)-1} \\
& \leq C(1+K)^{n / m}(1+|\lambda|)^{(n / m)-1},
\end{aligned}
$$

as claimed.

\section{The Solution $\zeta_{0}^{*}(Y ; \lambda)$ of the Equation $\zeta=G(\zeta ; Y ; \lambda)$}

We know that $A^{-1}\langle x\rangle^{-1} \leq \zeta_{0}(\varsigma, x)=q_{\psi}(x, \varsigma)^{-1} \leq A\langle x\rangle^{-1}$, $Y=(\varsigma, x) \in \widetilde{S}_{Y}=\mathbb{S}^{n-1} \times\left\{x \in \mathbb{R}^{n}:\langle x\rangle \leq \varkappa \lambda\right\}$, and that 
$k_{1}>4 A C>2 A C>2$. Moreover, $k_{2}>1$ is chosen so large that, in particular, on $\operatorname{supp} U_{2} \supset \operatorname{supp} V_{2}$, the absolute value of the $\zeta$-derivative of $G$ is less than $k_{0} \leq 1$, uniformly with respect to $Y \in \widetilde{S}_{Y}, \lambda \geq \lambda_{0},(X, Y ; \lambda) \in \operatorname{supp} V_{2}$. We want to show that once $k_{1}$ is fixed, the choice of such a suitably large $k_{2}>1$ allows to make $G$ a contraction on the compact set $I_{x}=$ $\left[A^{-1}(1-(\varepsilon / 2))\langle x\rangle^{-1}, A(1+(\varepsilon / 2))\langle x\rangle^{-1}\right] \subset\left[c^{-1}\langle x\rangle^{-1}, c\langle x\rangle^{-1}\right]$, uniformly with respect to $(\varsigma, x), \lambda \geq \lambda_{0}$, provided $\langle x\rangle \leq \varkappa \lambda$, $\varkappa=(1-(\varepsilon / 2))\left[A\left(2 k_{2}\right)^{m}\right]^{-1}$. This gives the existence and unicity of $\zeta_{0}^{*}(Y ; \lambda) \in I_{x}$ such that $X_{0}^{*}(Y ; \lambda)=\left(0, \zeta_{0}^{*}(Y ; \lambda)\right)$ is the unique stationary point of $F_{2}(X ; Y ; \lambda)$, with respect to $X$, which belongs to the support of $V_{2}(X ; Y ; \lambda)$ for $\langle x\rangle \leq u \lambda$.

First of all, the presence of the factors $H_{1}(\langle x\rangle$ $\left.\left\langle(\lambda \zeta)^{1 / m} \varsigma\right\rangle^{m}\right) / \lambda$ and $H_{2}\left((\lambda \zeta)^{1 / m}\right)$ in the expression of $U_{2}$ implies $(\lambda \zeta)^{1 / m} \geq k_{2} \Rightarrow\left\langle(\lambda \zeta)^{1 / m}\right\rangle \leq\left(1+k_{2}^{-2}\right)^{1 / 2}(\lambda \zeta)^{1 / m}$ and

$$
\begin{aligned}
\left(2 k_{1}\right)^{-1} & \leq \frac{\langle x\rangle\left\langle(\lambda \zeta)^{1 / m} \varsigma\right\rangle^{m}}{\lambda} \\
& \leq\langle x\rangle\left(1+k_{2}^{-2}\right)^{m / 2} \zeta \\
& \Longrightarrow\left[2 k_{1}\left(1+k_{2}^{-2}\right)^{m / 2}\right]^{-1} \\
& \leq\langle x\rangle \zeta<\frac{\langle x\rangle\left\langle(\lambda \zeta)^{1 / m} \varsigma\right\rangle^{m}}{\lambda} \leq 2 k_{1} \\
& \Longrightarrow \zeta \in\left[c^{-1}\langle x\rangle^{-1}, c\langle x\rangle^{-1}\right], \\
c & =2 k_{1}\left(k_{2}^{-2}+1\right)^{m / 2} .
\end{aligned}
$$

Since $k_{1}>4 A C>2 A C$, clearly $I_{x} \subset\left[c^{-1}\langle x\rangle^{-1}, c\langle x\rangle^{-1}\right]$. With an arbitrarily chosen $\varepsilon \in(0,1 / 2)$, take $k_{2}>\max \{B, 1\}$ such that $\lambda \zeta>k_{2}^{m}$ implies $\left|S^{-1,0}\left(x,(\lambda \zeta)^{1 / m}\right)\right| \leq \varepsilon / 2$ and $\left|\zeta_{0} \zeta^{-1} S^{-1,0}\left(x,(\lambda \zeta)^{1 / m}\right)\right| \leq k_{0}<1$, which is possible, in view of (3) and of the fact that $\zeta_{0} \zeta^{-1}$ is bounded on supp $V_{2}$. Fix $\lambda \geq \lambda_{0}>2 k_{1}\left\langle 2 k_{2}\right\rangle^{m}$ and $\langle x\rangle \leq \varkappa \lambda$. Then, on $\operatorname{supp} V_{2}$,

$$
\begin{aligned}
\zeta \in I_{x} & \Longrightarrow \lambda \zeta>\left(1-\frac{\varepsilon}{2}\right)^{-1} A\left(2 k_{2}\right)^{m}\langle x\rangle A^{-1}\left(1-\frac{\varepsilon}{2}\right)\langle x\rangle^{-1} \\
& =\left(2 k_{2}\right)^{m}>k_{2}^{m} \\
& \Longrightarrow G(\zeta ; Y ; \lambda)=\zeta_{0}\left(1+S^{-1,0}\left(x,(\lambda \zeta)^{1 / m}\right)\right) \\
& \in\left[A^{-1}\left(1-\frac{\varepsilon}{2}\right)\langle x\rangle^{-1}, A\left(1+\frac{\varepsilon}{2}\right)\langle x\rangle^{-1}\right]=I_{x} \\
& \Longleftrightarrow G(\cdot ; Y ; \lambda): I_{x} \longrightarrow I_{x} .
\end{aligned}
$$

Since $\left|\partial_{\zeta} G(\zeta ; Y ; \lambda)\right|=\left|\zeta_{0} \zeta^{-1} S^{-1,0}\left(x,(\lambda \zeta)^{1 / m}\right)\right| \leq k_{0}<1$, for all $\zeta \in I_{x},\langle x\rangle \leq \varkappa \lambda$, we have proved that for any choice of $Y \in \widetilde{S}_{Y}, \lambda \geq \lambda_{0}$ as above, $G(\cdot ; Y ; \lambda)$ has a unique fixed point in $\zeta_{0}^{*}=\zeta_{0}^{*}(Y ; \lambda) \in I_{x}$, solution of $\zeta=G(\zeta ; Y ; \lambda)$.

By well-known corollaries of the fixed point theorem for strict contractions on compact subsets of metric spaces, we of course have that $\zeta_{0}^{*}$ depends smoothly on $Y$ and $\lambda$. Moreover, since $\zeta_{0}^{*} \in I_{x}$ for all $Y \in \widetilde{S}_{Y}, \lambda \geq \lambda_{0}$, obviously $\zeta_{0}^{*} \sim\langle x\rangle^{-1}$ and

$$
\begin{aligned}
\zeta_{0}^{*}(Y ; \lambda) & =\zeta_{0}\left(1+S^{-1,0}\left(x,\left(\lambda \zeta_{0}^{*}(Y ; \lambda)\right)^{1 / m}\right)\right) \\
& \longrightarrow \zeta_{0}(\varsigma, x), \quad \lambda \longrightarrow+\infty,
\end{aligned}
$$

pointwise for any $(\varsigma, x)$. Moreover, by the choices of $k_{1}, k_{2}$, and $\varepsilon$,

$$
\begin{gathered}
\frac{\langle x\rangle\left\langle\left(\lambda \zeta_{0}^{*}(\varsigma, x ; \lambda)\right)^{1 / m}\right\rangle^{m}}{\lambda}=\left[\frac{\langle x\rangle^{2 / m}}{\lambda^{2 / m}}+\left(\langle x\rangle \zeta_{0}^{*}\right)^{2 / m}\right]^{m / 2} \\
>A^{-1}\left(1-\frac{\varepsilon}{2}\right)>k_{1}^{-1}, \\
\frac{\langle x\rangle\left\langle\left(\lambda \zeta_{0}^{*}(\varsigma, x ; \lambda)\right)^{1 / m}\right\rangle^{m}}{\lambda} \\
\left\langle\left[\varkappa^{2 / m}+\left(A\left(1+\frac{\varepsilon}{2}\right)\right)^{2 / m}\right]^{m / 2}\right. \\
=A\left[\left(1-\frac{\varepsilon}{2}\right)^{2 / m} A^{-4 / m}\left(2 k_{2}\right)^{-2}+\left(1+\frac{\varepsilon}{2}\right)^{2 / m}\right]^{m / 2}<k_{1}, \\
\langle x\rangle \leq \varkappa \lambda \Longleftrightarrow \lambda A^{-1}\left(1-\frac{\varepsilon}{2}\right)\langle x\rangle^{-1} \geq\left(2 k_{2}\right)^{m} \\
\Longrightarrow \lambda \zeta_{0}^{*}(\varsigma, x ; \lambda) \in\left[\left(2 k_{2}\right)^{m},+\infty\right) .
\end{gathered}
$$

These imply, for any $\varsigma \in \mathbb{S}^{n-1}, x \in \mathbb{R}^{n}, \lambda \geq \lambda_{0}$ such that $\langle x\rangle \leq x \lambda$,

$$
\begin{gathered}
H_{1}\left(\frac{\langle x\rangle\left\langle\left(\lambda \zeta_{0}^{*}(\varsigma, x ; \lambda)\right)^{1 / m}\right\rangle^{m}}{\lambda}\right)=1, \\
1-H_{2}\left(\left(\lambda \zeta_{0}^{*}(\varsigma, x ; \lambda)\right)^{1 / m}\right)=1 .
\end{gathered}
$$

Of course, by the choice of $H_{3}$, for $Y \in \widetilde{S}_{Y}, \lambda \geq \lambda_{0}$,

$$
\zeta_{0}^{*} \in I_{x} \Longrightarrow H_{3}\left[\frac{\zeta_{0}^{*}(\varsigma, x ; \lambda)}{\zeta_{0}(\varsigma, x)}-1\right]=1
$$

\section{Acknowledgments}

The authors wish to thank U. Battisti, L. Rodino, and E. Schrohe for useful discussions and hints. Thanks are also due to N. Batavia. The first author was partially supported by the PRIN Project "Operatori Pseudo-Differenziali ed Analisi Tempo-Frequenza" (Director of the national project: G. Zampieri; local supervisor at Università di Torino: L. Rodino). The first author also gratefully acknowledges the support by the Institut für Analysis, Fakultät für Mathematik und Physik, Gottfried Wilhelm Leibniz Universität Hannover, during his stay as Visiting Scientist in the Academic Year 2011/2012, where this paper was partly developed and completed. 


\section{References}

[1] H. O. Cordes, The Technique of Pseudodifferential Operators, vol. 202, Cambridge University Press, Cambridge, Mass, USA, 1995.

[2] C. Parenti, "Operatori pseudo-differenziali in $\mathbb{R}^{n}$ e applicazioni," Annali di Matematica Pura ed Applicata, vol. 93, pp. 359-389, 1972.

[3] R. B. Melrose, Geometric Scattering Theory, Stanford Lectures, Cambridge University Press, Cambridge, Mass, USA, 1995.

[4] M. A. Shubin, Pseudodifferential Operators and Spectral Theory, Springer Series in Soviet Mathematics, Springer, Berlin, Germany, 1987.

[5] E. Schrohe, "Spaces of weighted symbols and weighted sobolev spaces on manifolds," in Pseudodifferential Operators (Oberwolfach, 1986), vol. 1256 of Lecture Notes in Mathematics, pp. 360377, Springer, Berlin, Germany, 1987.

[6] Y. V. Egorov and B.-W. Schulze, Pseudo-differential operators, singularities, applications, vol. 93, Birkhäuser Verlag, Basel, Switzerland, 1997.

[7] L. Maniccia and P. Panarese, "Eigenvalue asymptotics for a class of md-elliptic $\psi$ do's on manifolds with cylindrical exits," Annali di Matematica Pura ed Applicata, vol. 181, no. 3, pp. 283-308, 2002.

[8] V. Ivrii, Microlocal Analysis and Precise Spectral Asymptotics, Springer, Berlin, Germany, 1998.

[9] L. Hörmander, "The spectral function of an elliptic operator," Acta Mathematica, vol. 121, no. 1, pp. 193-218, 1968.

[10] V. Guillemin, "A new proof of Weyl's formula on the asymptotic distribution of eigenvalues," Advances in Mathematics, vol. 55, no. 2, pp. 131-160, 1985.

[11] H. Kumano-go, Pseudodifferential Operators, MIT Press, Boston, Mass, USA, 1981.

[12] P. Boggiatto, E. Buzano, and L. Rodino, Global Hypoellipticity and Spectral Theory, vol. 92, Akademie Verlag, Berlin, Germany, 1996.

[13] B. Helffer, Théorie Spectrale Pour Des Opérateurs Globalement Elliptiques, vol. 112, Astérisque, 1984.

[14] L. Hörmander, "On the asymptotic distribution of the eigenvalues of pseudodifferential operators in $\mathbb{R}^{n}$," Arkiv för Matematik, vol. 17, no. 2, pp. 297-313, 1979.

[15] A. Mohammed, "Comportement asymptotique, avec estimation du reste, des valor propres d'une classe d'operateurs pseudodifferentiels sur $\mathbb{R}^{n,}$, Mathematische Nachrichten, vol. 14, pp. 127-186, 1989.

[16] F. Nicola, "Trace functionals for a class of pseudo-differential operators in $\mathbb{R}^{n}$, " Mathematical Physics, Analysis and Geometry, vol. 6, no. 1, pp. 89-105, 2003.

[17] T. Christiansen and M. Zworski, "Spectral asymptotics for manifolds with cylindrical ends," Annales de l'Institut Fourier, vol. 45, no. 1, pp. 251-263, 1995.

[18] U. Battisti and S. Coriasco, "Wodzicki residue for operators on manifolds with cylindrical ends," Annals of Global Analysis and Geometry, vol. 40, no. 2, pp. 223-249, 2011.

[19] L. Maniccia, E. Schrohe, and J. Seiler, "Complex powers of classical SG-pseudodifferential operators," Annali dell'Universitá di Ferrara, vol. 52, no. 2, pp. 353-369, 2006.

[20] M. Borsero and S. Coriasco, "Eigenvalue asymptotics of Schrödinger-type operators on manifolds with ends," In Preparation.

[21] M. Borsero, Microlocal analysis and spectral theory of elliptic operators on non-compact manifolds [thesis], Tesi di Laurea Magistrale in Matematica, Universitá di Torino, 2011.
[22] S. Coriasco, "Fourier integral operators in SG classes. I. Composition theorems and action on SG sobolev spaces," Rendiconti del Seminario Matematico. Università e Politecnico di Torino, vol. 57, no. 4, pp. 249-302, 1999.

[23] S. Coriasco, "Fourier integral operators in SG classes. II. Application to SG hyperbolic cauchy problems," Annali dell'Università di Ferrara, vol. 44, pp. 81-122, 1998.

[24] S. Coriasco and L. Rodino, "Cauchy problem for SG-hyperbolic equations with constant multiplicities," Ricerche di Matematica, vol. 48, Supplement, pp. 25-43, 1999.

[25] A. Grigis and J. Sjöstrand, Microlocal Analysis for Differential Operators, vol. 196, Cambridge University Press, Cambridge, Mass, USA, 1994.

[26] B. Helffer and D. Robert, "Propriétés asymptotiques du spectre d'opérateurs pseudodifférentiels sur $\mathbb{R}^{n}$," Communications in Partial Differential Equations, vol. 7, no. 7, pp. 795-882, 1982.

[27] H. Tamura, "Asymptotic formulas with sharp remainder estimates for eigenvalues of elliptic operators of second order," Duke Mathematical Journal, vol. 49, no. 1, pp. 87-119, 1982.

[28] S. Coriasco and P. Panarese, "Fourier integral operators defined by classical symbols with exit behaviour," Mathematische Nachrichten, vol. 242, pp. 61-78, 2002.

[29] S. Coriasco and L. Maniccia, "Wave front set at infinity and hyperbolic linear operators with multiple characteristics," Annals of Global Analysis and Geometry, vol. 24, no. 4, pp. 375400, 2003.

[30] J. J. Duistermaat, Fourier Integral Operators, vol. 130 of Progress in Mathematics, Birkhäuser, Boston, Mass, USA, 1996.

[31] L. H. Hörmander, The Analysis of Linear Partial Differential Operators I-IV. Classics in Mathematics, Springer, Berlin, Germany, 2009.

[32] B. Helffer and D. Robert, "Comportement asymptotique précisé du spectre d'opérateurs globalement elliptiques dans $\mathbb{R}^{n}$, " Comptes Rendus de l'Académie des Sciences. Série I. Mathématique, vol. 292, no. 6, pp. 363-366, 1981.

[33] F. Nicola and L. Rodino, "SG pseudo-differential operators and weak hyperbolicity," Pliska Studia Mathematica Bulgarica, vol. 15, pp. 5-20, 2003. 


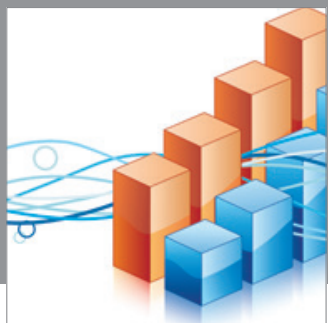

Advances in

Operations Research

mansans

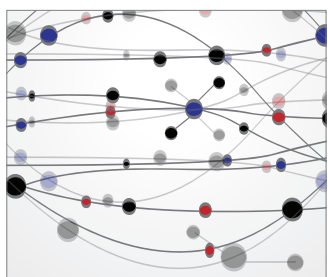

The Scientific World Journal
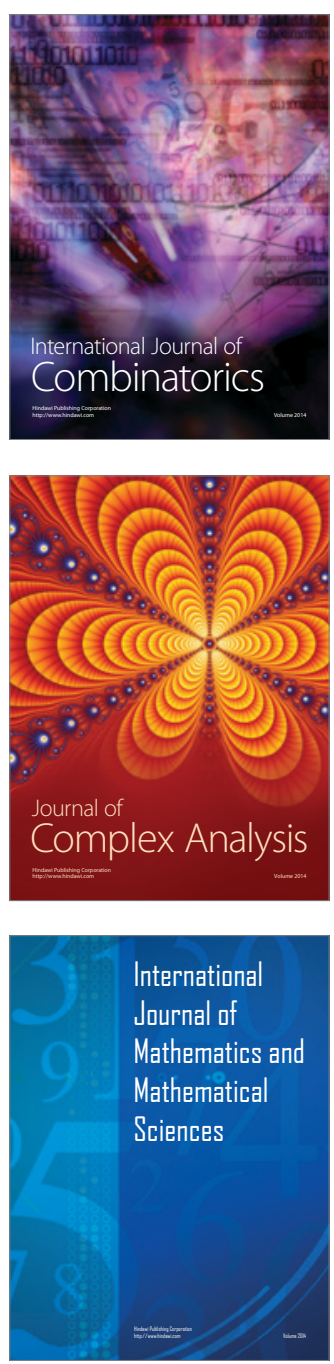
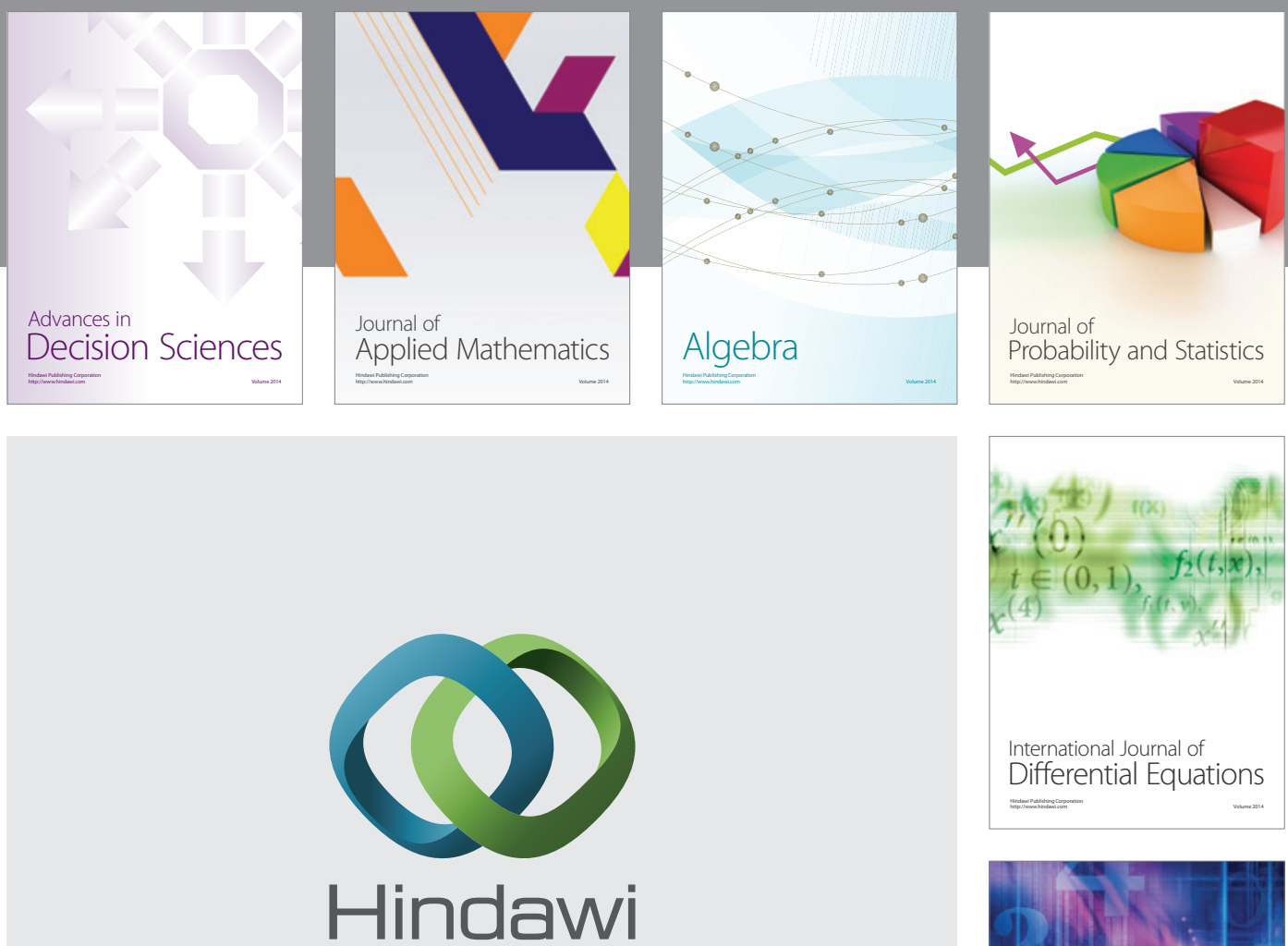

Submit your manuscripts at http://www.hindawi.com
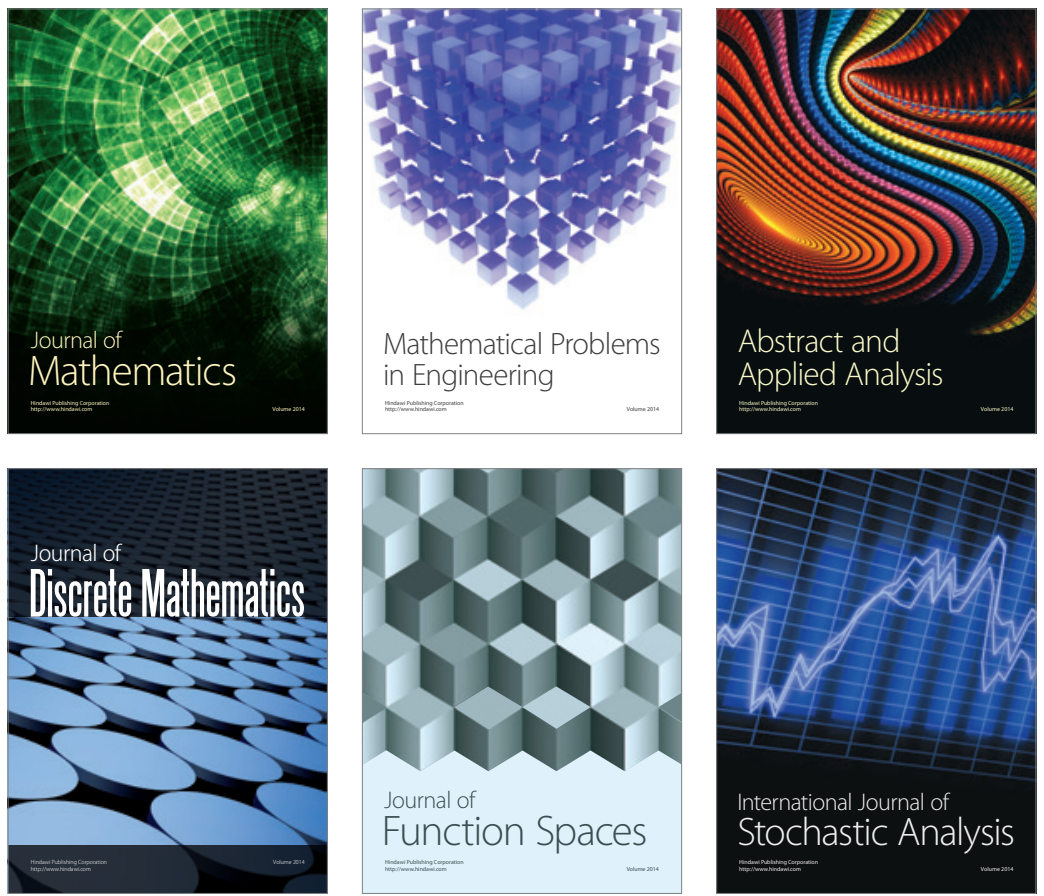

Journal of

Function Spaces

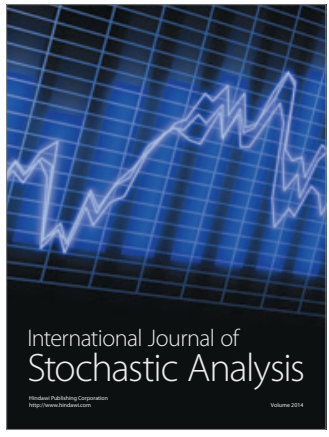

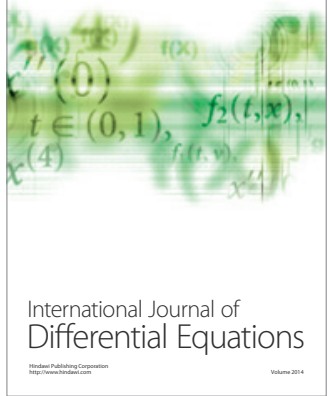
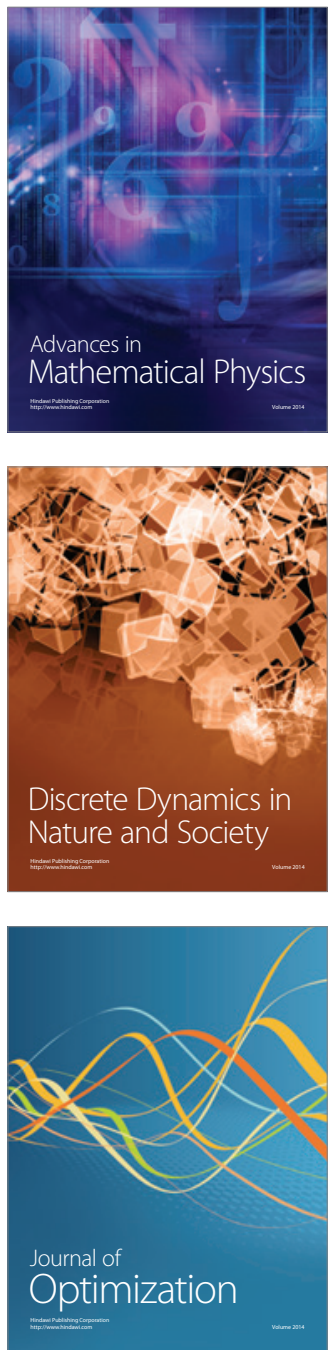